\title{
Numerical Investigation of a Bluff-Body Stabilised Nonpremixed Flame with Differential Reynolds-Stress Models
}

\author{
GUOXIU LI ${ }^{1}$, BERTRAND NAUD ${ }^{2}$ and DIRK ROEKAERTS ${ }^{3, \star}$ \\ ${ }^{1}$ School of Mechanical, Electronic and Control Engineering, Northern Jiaotong University, \\ Beijing 100044, P.R. China \\ ${ }^{2}$ LITEC, Consejo Superior de Investigaciones Científicas, María de Luna 10, 50018 Zaragoza, \\ Spain \\ ${ }^{3}$ Thermal and Fluids Sciences, Delft University of Technology, Lorentzweg 1, 2628 CJ Delft, \\ The Netherlands
}

Received 17 September 2002; accepted in revised form 7 July 2003

\begin{abstract}
A numerical investigation of a bluff-body stabilised nonpremixed flame, and the corresponding nonreacting flow, has been performed with differential Reynolds-stress models (DRSMs). The equilibrium chemistry model is employed and an assumed-shape beta function PDF approach is used to represent the interaction between turbulence and chemistry. The Reynolds flux of the mixture fraction is obtained from a transport equation, hence a full second moment closure is used. To clarify the applicability of the existing DRSMs in this complex flame, several models, including LRR-IP model, JM model, SSG model as well as a modified LRR-IP model, have been applied and evaluated. The existing models, with default values of the coefficients, cannot provide overall satisfactory predictions for this challenging test case. The standard LRR-IP model over predicts the centreline velocity decay rate, and therefore does not perform satisfactory. The modified LRR-IP model, with model constant $C_{\epsilon 1}=1.6$ instead of the standard value 1.44 (here named BM-M1), gives better results for the mean velocity. However in the nonreacting case this does not lead to improvement in predicting rms fluctuating velocities especially downstream of the recirculation zone. Motivated by the need to improve the prediction, a new modification of the LRR-IP model is proposed (BM$\mathrm{M} 2$ ), with model constant $C_{2}=0.7$ in the pressure strain correlation rather than the standard value 0.6 . With the new modified model, a very significant improvement of the prediction of flow field is obtained in the nonreacting case, whereas in the reacting case the prediction of the flow field is of the same overall quality as with BM-M1. This shows that some DRSMs have different behaviour in the nonreacting case and the reacting case. In the reacting case also the mean and variance of mixture fraction are considered and it is found that the best results are obtained with the BM-M1 model, with SSG as second best. Combining the results for flow field and mixture fraction field it is concluded that the BM-M1 model is recommended for further studies of this bluff-body stabilised flame. Grid independence of the result is demonstrated.
\end{abstract}

Key words: bluff-body flame, differential Reynolds-stress model, turbulence modelling, turbulent combustion.

\footnotetext{
^ Author for correspondence; E-mail address: dirkr@ws.tn.tudelft.nl.
} 


\section{Introduction}

The bluff-body stabilised flame has received special attention and been widely studied recently $[3,4,8,9,12,13,19,20,28,29]$. In addition to its practical interest, the bluff-body flame is a very challenging test for turbulence models as well as chemistry models. The flame is stabilised behind the bluff-body by the intense mixing of fuel and air and by the hot products providing a continuous ignition source in the recirculation zone [3]. In the resulting flow field, the central fuel jet is separated from the coflowing air stream by the hot recirculation zone in the wake of the bluff-body.

The bluff-body stabilised flame investigated here was one of the target flames in the International Workshop on Measurement and Computation of Turbulent Nonpremixed Flames (TNF workshop) [15, 17]. It was studied experimentally by Dally et al. [3, 4]. Dally et al. [3] reported simulation results obtained using standard and modified $k-\epsilon$ model and Reynolds-stress models. They found that a simple modification to the $C_{\epsilon 1}$ constant in the dissipation equation gave better prediction results in the recirculation zone, but did not lead to any improvement further downstream, especially for rms fluctuating, axial and radial velocities. Merci et al. [19] applied a new cubic nonlinear eddy viscosity turbulence model that provided better prediction results in the recirculation zone compared with the standard linear models. But it is noteworthy that all models employed in [19] underestimate the persistence of the jet, which means that the minimum value of the velocity on the symmetry axis is underpredicted especially further downstream. The same flame has also been modelled with a conditional moment closure (CMC) model by Kim et al. $[12,13]$ and with a coupled radiation/flamelet combustion model by Hossain et al. [8], and also with Monte Carlo PDF models by Jenny et al. [9] and Muradoglu et al. [20] more recently. A similar bluff-body stabilised flame with different geometrical configuration and fuel has also been studied numerically by Wouters et al. [28-30] using $k-\epsilon$ model and Reynolds-stress models as well as hybrid finite volume/Monte Carlo PDF model.

It is noteworthy that almost all of the approaches mentioned above can give a good prediction in the recirculation zone, but only a poor prediction with a pronounced discrepancy, at least not small, from measurements further downstream. This is quite remarkable and needs further study and improvement.

The objective of this work is to explore whether significant improvements in the prediction of flow field characteristics (mean velocity and Reynolds stress) and the overall mixing characteristics (mean and variance of mixture fraction) can be obtained by improving the turbulence model. For this purpose the chemistry model and the turbulence-chemistry interaction model can be kept simple, provided the limitations are kept in mind. We shall use equilibrium chemistry in the framework of an assumed beta function shape of the PDF of mixture fraction [22].

Staying in the framework of the Reynolds averaged Navier-Stokes (RANS) approach the next logical step beyond eddy-viscosity models (EVMs) are differ- 
ential Reynolds-stress models (DRSMs) which solve the Reynolds stresses from their respective transport equations [7]. The superiority of DRSMs has already been demonstrated by a lot of computational examples [7] and also, they form a counterpart of Langevin models in the hybrid finite volume/Monte Carlo PDF method for reacting flow [24]. For these two reasons they are selected.

So far in the literature a number of differential Reynolds-stress models have been proposed. A major difference between the various DRSMs is in the treatment of the pressure strain term. Among those models, the so-called "Basic Model", consisting of the Rotta model for the slow part and the Isotropisation-of-Production (IP) model for the rapid part, is widely used. It was proposed by Launder et al. [14], and is also referred to as LRR-IP model. In addition to the LRR-IP model, many other well-known DRSMs have been proposed, including: the original model of Hanjalic and Launder (HL) [6]; the quasi-isotropic model (LRR-QI) of Launder et al. [14]; the model proposed by Jones and Musonge (JM) [10]; the model of Fu, Launder and Tselepidakis (FLT) [5]; the model of Craft and Launder (CL) [1]; and the SSG model proposed by Speziale, Sarkar, and Gatski [27].

Considering that until now little research has been conducted to numerically investigate the complicated and challenging bluff-body stabilised flame with the above-mentioned DRSMs, it is worthwhile to clarify the applicability of the various DRSMs to this complex flow. In the present study several typical models including LRR-IP model, JM model, SSG model as well as a modified LRR-IP model have been applied and evaluated in the bluff-body flame. A new modification of the LRR-IP model is proposed to demonstrate that by varying model constants it is possible to improve the predictions of the bluff-body flame in an adequate way. The objective is of course not to propose a new generally applicable DRSM, but to develop a model that subsequently can be successfully exploited in further studies on turbulence-chemistry interaction in this and similar configurations. Hence in the present study we investigate and evaluate the performance of several typical DRSMs in predicting the bluff-body flame by comparison with the available measured data, and make a modification in the LRR-IP model to improve the prediction results. Special attention is paid to demonstration of grid-independence of the results.

The step from eddy viscosity model to DRSM in the modelling of the turbulent velocity field has a counterpart in the modelling of the turbulent mixture fraction field, namely the step from gradient diffusion approximation of the Reynolds flux to differential equation for the Reynolds flux. Here the differential Reynoldsflux model will be used. Then presumably the results obtained are also of direct relevance for hybrid finite volume/Monte Carlo velocity-scalar PDF methods, because of the close connection of the Reynolds-flux model in that approach to the Reynolds-flux transport equation [24, 30]. 


\section{Turbulence Models}

\subsection{REYNOLDS-STRESS EQUATIONS}

In the differential Reynolds-stress model, the Reynolds stresses are calculated from their own transport equations. Assuming high Reynolds number, viscous terms are neglected except for the viscous dissipation term $\epsilon_{i j}$. The Reynolds-stress equation for variable density flows then reads $[11,26,30]$ :

$$
\begin{aligned}
& \bar{\rho} \frac{D u_{i}^{\prime \prime \prime} u_{j}^{\prime \prime}}{D t}=-\bar{\rho} \widetilde{u}_{i}^{\prime \prime} u_{k}^{\prime \prime} \frac{\partial \widetilde{U}_{j}}{\partial x_{k}}-\bar{\rho} u_{j}^{\prime \prime} u_{k}^{\prime \prime} \frac{\partial \widetilde{U}_{i}}{\partial x_{k}} \\
& -\left[\overline{u_{i}^{\prime \prime} \frac{\partial p^{\prime}}{\partial x_{j}}}+\overline{u_{j}^{\prime \prime} \frac{\partial p^{\prime}}{\partial x_{i}}}-\frac{2}{3} \delta_{i j} \overline{u_{k}^{\prime \prime} \frac{\partial p^{\prime}}{\partial x_{k}}}\right] \\
& -\bar{\rho} \epsilon_{i j} \\
& -\frac{\partial}{\partial x_{k}}\left[\bar{\rho} \widetilde{u_{i}^{\prime \prime} u_{j}^{\prime \prime} u_{k}^{\prime \prime}}+\frac{2}{3} \delta_{i j} \overline{u_{k}^{\prime \prime} p^{\prime}}\right] \\
& -\overline{u_{i}^{\prime \prime}} \frac{\partial \bar{p}}{\partial x_{j}}-\overline{u_{j}^{\prime \prime}} \frac{\partial \bar{p}}{\partial x_{i}} \\
& +\frac{2}{3} \delta i j \overline{p^{\prime} \frac{\partial u_{k}^{\prime \prime}}{\partial x_{k}}} \text {. }
\end{aligned}
$$

Here, the overbar denotes the Reynolds average and the tilde identifies Favreaveraged quantities. The terms on the RHS are: (1a) the production by mean shear $P_{i j}$, (1b) the pressure-strain correlation $\Phi_{i j}$, (1c) the viscous dissipation $\epsilon_{i j}$, (1d) the turbulent flux $\Gamma_{i j}$ and two terms which are zero in constant density flows containing (1e) a mean pressure gradient, and (1f) the trace of the fluctuating strain tensor.

- The production term $P_{i j}$ is in closed form and therefore does not need to be modelled, whereas the pressure-strain correlation, dissipation, turbulent flux, and fluctuating density terms have to be modelled.

- The diffusive transport is described by the gradient transport approximation [2],

$$
\widetilde{u_{i}^{\prime \prime} u_{j}^{\prime \prime} u_{k}^{\prime \prime}}=-C_{s} \frac{2}{3} \widetilde{u_{k}^{\prime \prime} u_{l}^{\prime \prime}} \frac{\partial \widetilde{u}_{i}^{\prime \prime} u_{j}^{\prime \prime}}{\partial x_{l}}
$$

where the constant $C_{s}$ is give in Table I.

- The viscous dissipation $\epsilon_{i j}$ is modelled by assuming local isotropy at the smallest scales where viscous dissipation takes place. The dissipation model is then defined as

$$
\epsilon_{i j}=\frac{2}{3} \epsilon \delta_{i j} .
$$


The turbulent energy dissipation rate $\epsilon$ is calculated from the following modelled transport equation [6]:

$$
\bar{\rho} \frac{D \epsilon}{D t}=C_{\epsilon} \frac{\partial}{\partial x_{k}}\left[\bar{\rho} \frac{k}{\epsilon} u_{k}^{\pi \prime \prime} u_{l}^{\prime \prime} \frac{\partial \epsilon}{\partial x_{l}}\right]+C_{\epsilon 1} \frac{\epsilon}{k} \bar{\rho} P_{k}-C_{\epsilon 2} \bar{\rho} \frac{\epsilon^{2}}{k}
$$

with $P_{k}$ the production of turbulent kinetic energy by mean shear given by

$$
P_{k}=-\widetilde{u_{i}^{\prime \prime} u_{j}^{\prime \prime}} \frac{\partial \widetilde{U}_{i}}{\partial x_{j}}
$$

The values of the constants used in the model are shown in Table I.

- Modelling of the fluctuating density terms can be found in [11].

- The final unclosed term is the pressure-strain correlation term, which has been the focal point of Reynolds-stress modelling. This term does not produce or destroy turbulent kinetic energy but only redistributes energy over the components of the stress tensor [28].

For constant density homogeneous flows with uniform mean velocity gradients, generally the pressure-strain correlation $\Phi_{i j}$ can be decomposed into a slow part and a rapid part according to the following expression [7, 25]:

$$
\Phi_{i j}=\Phi_{i j, 1}+\Phi_{i j, 2} .
$$

Generally the first item $\Phi_{i j, 1}$, which is called the slow pressure-strain term, contains only the velocity fluctuations and causes the turbulence to approach an isotropic state. It is also called the "return to isotropy" term and is normally modelled in terms of stress anisotropy tensor and its first and/or second invariants [31]. The second term $\Phi_{i j, 2}$ is referred to as the rapid pressure-strain because it immediately responds to change in the mean velocity gradients. This term is also often called the "isotropisation of production" term. It can be modelled in terms of the mean rate of strain, mean vorticity and stress anisotropy tensor. The pressure-strain correlation models used in the present study are described as follows.

\subsubsection{LRR-IP Model}

A basic pressure-strain model, represented by a combination of a linear returnto-isotropy or Rotta term and a linear isotropisation-of-production (IP) term, was proposed by Launder et al. [14], and is also referred to as LRR-IP model, the slow part and rapid part are modelled respectively by

$$
\begin{aligned}
& \Phi_{i j, 1}=-C_{1} \bar{\rho} \epsilon a_{i j}, \\
& \Phi_{i j, 2}=-C_{2}\left(P_{i j}-\frac{1}{3} P_{k k} \delta_{i j}\right),
\end{aligned}
$$


where $C_{1}$ and $C_{2}$ are model constants which are given in Table I, $\delta_{i j}$ the Kronecker tensor. The stress anisotropy tensor $a_{i j}$ is defined as

$$
a_{i j}=\frac{\widetilde{u_{i}^{\prime \prime} u_{j}^{\prime \prime}}}{k}-\frac{2}{3} \delta_{i j},
$$

where $k$ is the turbulent kinetic energy, and $P_{i j}$ is defined by

$$
P_{i j}=-\bar{\rho} u_{i}^{\prime \prime} u_{k}^{\prime \prime} \frac{\partial \widetilde{U}_{j}}{\partial x_{k}}-\bar{\rho} u_{j}^{\prime \prime} u_{k}^{\prime \prime} \frac{\partial \widetilde{U}_{i}}{\partial x_{k}} .
$$

A third contribution to the pressure strain term in the LRR-IP model, namely the wall reflection term [14] was also included.

\subsubsection{JM Model}

In turbulent reacting flows, strong density variations may occur and the trace of the strain tensor is not necessarily zero. The model by Jones and Musonge is considered here because it is developed specially for variable density flows. For the slow part, as for the LRR-IP model, the Rotta term shown in Equation (7) is employed (with $C_{1}=3.0$ ). The rapid part of the model is

$$
\begin{aligned}
\Phi_{i j, 2}= & \frac{3}{2} C_{2}\left(D_{i j}-\frac{1}{3} P_{k k} \delta_{i j}\right)+C_{3}\left(D_{i j}-P_{i j}\right) \\
& +C_{4} \bar{\rho} k\left(\frac{\partial \widetilde{U}_{i}}{\partial x_{j}}+\frac{\partial \widetilde{U}_{j}}{\partial x_{i}}-\frac{2}{3} \frac{\partial \widetilde{U}_{k}}{\partial x_{k}} \delta_{i j}\right)+C_{5} \bar{\rho} k a_{i j} \frac{\partial \widetilde{U}_{k}}{\partial x_{k}}
\end{aligned}
$$

where

$$
D_{i j}=-\bar{\rho} u_{i}^{\prime \prime} u_{k}^{\prime \prime} \frac{\partial \widetilde{U}_{k}}{\partial x_{j}}-\bar{\rho} u_{j}^{\prime \prime} u_{k}^{\prime \prime} \frac{\partial \widetilde{U}_{k}}{\partial x_{i}} .
$$

This model has been applied successfully to a wide range of flows including confined swirling flows with and without density variations. The values of the model constants used here are the optimal values reported by Jones [11] in 1994, namely: $C_{1}=-3.0, C_{2}=-0.44, C_{3}=0.46, C_{4}=-0.23$, as shown in Table I. The last term in Equation (11) which is zero in constant density flow may play a important role in turbulent flames. However, this term is discarded here by specifying $C_{5}=0$ since no value has been proposed for this constant.

\subsubsection{SSG Model}

In 1991, Speziale, Sarkar and Gatski analyzed the modelling of the pressure-strain correlation, compared various existing models and on the basis of invariance considerations along with dynamical systems theory proposed a quadratic nonlinear model called SSG model [27]. They found that the model performed better than 
Table I. Summary of values of model constants for different DRSM models. ${ }^{\star}$

\begin{tabular}{lccccccccccc}
\hline & $C_{1}$ & $C_{1 s}$ & $C_{2}$ & $C_{3}$ & $C_{3 s}$ & $C_{4}$ & $C_{5}$ & $C_{\epsilon}$ & $C_{\epsilon 1}$ & $C_{\epsilon 2}$ & $C_{s}$ \\
\hline LRR-IP & 1.8 & - & 0.6 & - & - & - & - & 0.18 & 1.44 & 1.92 & 0.22 \\
JM & 3.0 & - & -0.44 & 0.46 & - & -0.23 & 0.0 & 0.18 & 1.44 & 1.92 & 0.22 \\
SSG & 1.7 & 1.05 & 0.9 & 0.8 & -0.65 & 0.625 & 0.2 & 0.18 & 1.44 & 1.83 & 0.22 \\
\hline
\end{tabular}

other models for a variety of homogeneous turbulence flows, however some deficiencies still remained in case of some complex mean flows. The slow part of SSG model can be expressed by

$$
\Phi_{i j, 1}=-\bar{\rho} \epsilon\left[C_{1} a_{i j}-C_{1 s}\left(a_{i k} a_{k j}-\frac{1}{3} A_{2} \delta_{i j}\right)\right],
$$

where the term proportional to $C_{1 s}$ is the essential nonlinear term and $A_{2}$ is the first invariant of the anisotropy tensor $A_{2}=a_{i j} a_{j i}$.

The rapid part is written in terms of the mean rate of strain $S_{i j}$, mean vorticity $W_{i j}$, and stress anisotropy tensor $a_{i j}$ :

$$
\begin{aligned}
\Phi_{i j, 2}= & -C_{2} P_{k} \bar{\rho} a_{i j}+\left(C_{3}+C_{3 s} A_{2}^{1 / 2}\right) \bar{\rho} k S_{i j} \\
& +C_{4} \bar{\rho} k\left(a_{i k} S_{j k}+a_{j k} S_{i k}-\frac{2}{3} a_{k l} S_{k l} \delta_{i j}\right) \\
& +C_{5} \bar{\rho} k\left(a_{i k} W_{j k}+a_{j k} W_{i k}\right),
\end{aligned}
$$

where

$$
S_{i j}=\frac{1}{2}\left(\frac{\partial \widetilde{U}_{i}}{\partial x_{j}}+\frac{\partial \widetilde{U}_{j}}{\partial x_{i}}\right), \quad W_{i j}=\frac{1}{2}\left(\frac{\partial \widetilde{U}_{i}}{\partial x_{j}}-\frac{\partial \widetilde{U}_{j}}{\partial x_{i}}\right), \quad P_{k}=-u_{i}^{\prime \prime \prime} u_{j}^{\prime \prime} \frac{\partial \widetilde{U}_{i}}{\partial x_{j}} .
$$

The term proportional to $C_{3 s}$ was included to have for the rapid part the most general expression linear in the anisotropy tensor. It should be noted that in the SSG model in the equation for dissipation rate a value of $C_{\epsilon 2}=1.83$ is used here (as opposed to the standard value of 1.92) as suggested by Speziale et al. [27].

\subsection{REYNOLDS-FLUX AND SCALAR-VARIANCE EQUATIONS}

Assuming only one scalar variable, the equation for the turbulent scalar flux or Reynolds flux $\widetilde{u_{i}^{\prime \prime} \phi^{\prime \prime}}$ and for scalar variance $\widetilde{\phi^{\prime \prime} \phi^{\prime \prime}}$ respectively read [26]:

$$
\bar{\rho} \frac{\widetilde{D u_{i}^{\prime \prime} \phi^{\prime \prime}}}{D t}=\widetilde{\rho} \widetilde{u_{j}^{\prime \prime} \phi^{\prime \prime}} \frac{\partial \widetilde{U}_{j}}{\partial x_{k}}-\bar{\rho} \widetilde{u_{i}^{\prime \prime} u_{j}^{\prime \prime}} \frac{\partial \widetilde{\phi}}{\partial x_{j}}
$$

^ For a more detailed comparison of the three model expressions considered as linear combination of nondimensional, symmetric, deviatoric tensors, we refer to [25]. 


$$
\begin{aligned}
& -\overline{\phi^{\prime \prime} \frac{\partial p^{\prime}}{\partial x_{i}}} \\
& -\frac{\partial}{\partial x_{j}}\left[\bar{\rho} \widetilde{u_{i}^{\prime \prime} u_{j}^{\prime \prime} \phi^{\prime \prime}}\right] \\
& -\overline{\phi^{\prime \prime} \frac{\partial \bar{p}}{\partial x_{i}}} \\
& -\bar{\rho} u_{i}^{\overline{u^{\prime \prime} S(\phi)}},
\end{aligned}
$$

and

$$
\begin{aligned}
\bar{\rho} \frac{D \widetilde{\phi^{\prime \prime} \phi^{\prime \prime}}}{D t}= & \widetilde{\rho} \widetilde{u_{j}^{\prime \prime} \phi^{\prime \prime}} \frac{\partial \widetilde{\phi}}{\partial x_{j}} \\
& -\bar{\rho} \epsilon_{\phi} \\
& -\frac{\partial}{\partial x_{j}}\left[\bar{\rho} \widetilde{u_{i}^{\prime \prime} \phi^{\prime \prime} \phi^{\prime \prime}}\right] \\
& -2 \bar{\rho} \widetilde{\phi^{\prime \prime} S(\phi)} .
\end{aligned}
$$

The terms on the right-hand sides of these equations are, in analogy with the Reynolds-stress equations, the production terms (15a) and (16a), the pressure scrambling term $\Pi_{i}^{\phi}(15 b)$, the viscous dissipation of scalar variance $\epsilon_{\phi}(16 b)$, the turbulent fluxes (15c) and (16c) and an additional mean pressure-gradient term which is zero in constant density flows (15d). Furthermore, for a reacting scalar, unclosed reaction source terms (15e) and (16d) appear in the equations. In this work the equations (15) and (16) are used for a nonreacting scalar (mixture fraction). The triple correlations are closed by a generalised gradient diffusion assumption. The effects of fluctuating pressure on the turbulent scalar fluxes (pressure scrambling) were modelled following [11].

The dissipation rate of scalar variance is linked to the dissipation rate of mechanical energy by $\left(g \equiv \widetilde{\phi^{\prime \prime} \phi^{\prime \prime}}\right)$ :

$$
\omega_{\phi} \equiv \frac{\epsilon_{\phi}}{g}=C_{\phi} \omega \equiv C_{\phi} \frac{\epsilon}{k} .
$$

The empirical constant $C_{\phi}$ has the standard value 2 . Although the constant may vary through the flow it is reasonably constant in diffusion flames where the fluctuations in velocity and scalars are induced by the same process, namely the different velocities and concentrations of fuel and oxidiser streams. 


\section{Grid and Boundary Conditions}

\subsection{COMPUTATIONAL DOMAIN AND GRID}

The computational domain, with the symmetry axis as boundary, is $300 \mathrm{~mm}$ long in the axial direction and $150 \mathrm{~mm}$ wide in the radial direction. In the axial direction, the lower boundary of the computational domain is at the jet exit plane. The computational grid consisting of $160 \times 128$ cells is stretched in the radial direction as well as the axial direction. The number of cells (in the radial direction) located in the jet, bluff-body and coflow is 8,80 and 40 , respectively. We find that it is quite important to include enough cells in the wake of the bluff-body to obtain the grid independent solutions. Grid independence has been checked by comparing with predictions on a grid twice as fine in both directions $(320 \times 256$, with 160 cells in radial direction in the wake of the bluff body) for the nonreacting flow using the LRR-IP model.

The results shown in Figure 1 demonstrate the grid independence. As a side remark we point out that, generally speaking, worse agreement with experiment is obtained if a too coarse grid is used.

\subsection{BOUNDARY CONDITIONS}

In the present bluff-body geometry, predictions are quite sensitive to inlet boundary conditions, and it is important to apply accurate inflow profiles in order to get reliable predictions. Here, the boundary conditions are carefully determined as follows. The inflow and the boundary conditions are specified in the same way as for the cold bluff-body case studied by Jenny et al. [9]. At the jet and coflow regions of the boundary, the experimental data are used for the axial velocity of the coflow, and also the profiles of normal Reynolds stresses of the centre jet and coflow are obtained and calculated from the experimental data. The axial velocity in the central jet region, the shear Reynolds stress, and the dissipation rate are all calculated according to the formula provided in [9]. The results are found to be rather insensitive to the applied boundary condition at the bluff body face, either no-slip boundary condition, with standard wall functions or free slip boundary condition. Symmetry conditions are applied at the symmetry axis.

\section{Modification of the LRR-IP Model for the Bluff-Body Flow}

Previous works on this bluff-body flame $[17,19]$ illustrate that factors influencing the quality of the prediction of the flow fields deserve special attention. This relates to the grid independence and boundary conditions, already discussed above, but also to the choice of turbulence model, including optimisation of model constants. A detailed study of effects of turbulence-chemistry interaction can be made only when the predictions of the flow field, including fluctuations is sufficiently accurate and to reach this status the modification of model constants is considered a recom- 

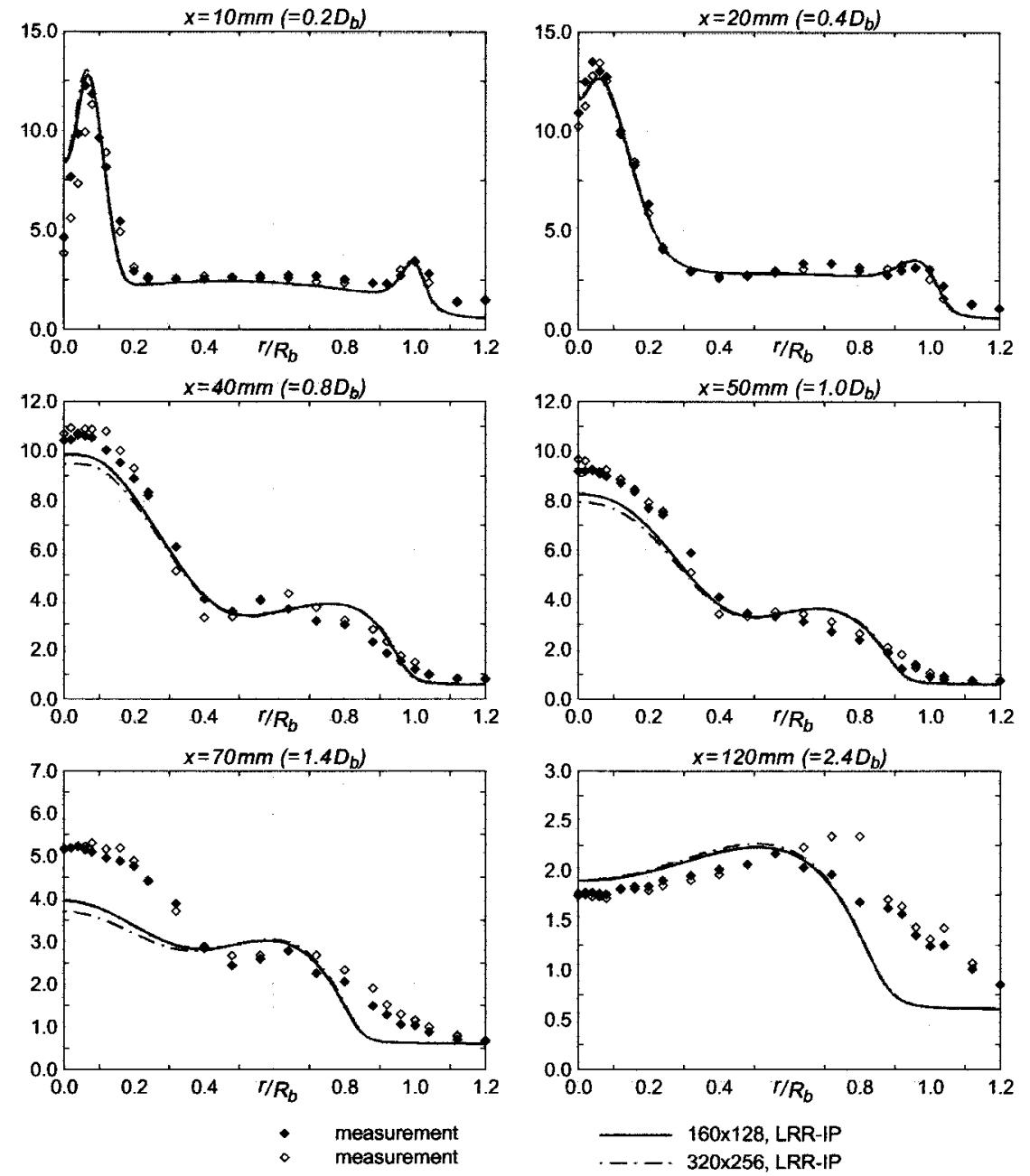

Figure 1. Radial profiles of rms fluctuation of axial velocity at different axial locations for the grids used to test grid independence (nonreacting flow, LRR-IP model).

mended procedure, provided choice of model constants is well-documented. Here we study the effect of modification of constants in the basic Reynolds-stress model (LRR-IP) but we do this as a complement to another approach that is to compare the relative quality of the predictions of the standard form of three well-established Reynolds-stress models (LRR-IP, JM, SSG).

It is well known that the standard Reynolds-stress model overpredicts the decay rate and the spreading rate of the round jet [23]. In the bluff-body flame, this point, already demonstrated by Dally et al. [3], is also confirmed in the present study. In order to fix this deficiency, some modifications for the model constants of the dissipation equation have been proposed as described in detail in [3]. In several 
modifications, the constants $C_{\epsilon 1}$ and $C_{\epsilon 2}$ (the constants in the dissipation equation) are made as a simple or complicated function of the velocity decay rate and jet width $[18,23]$. However despite a number of complicated modifications, Dally et al. [3] have shown that, with the use of constant value of $C_{\epsilon 1}=1.6$ instead of the standard value of 1.44 a very significant improvement was obtained in the calculation results for the round jets, better than any other complicated modifications (for convenience here we denote this modification of LRR-IP model as BM-M1, for "basic model modification 1"). However, for the bluff-body flame, except that the decay rate of the centreline velocity is correctly predicted in the recirculation zone, it did not lead to any improvement for rms fluctuating velocities especially further downstream. Motivated by this deficiency, we here propose a new modification of the LRR-IP model. Instead of modifying the model constant $C_{\epsilon 1}$ in the dissipation equation, we change the model constant $C_{2}$ in the pressure-strain model from 0.6 to 0.7 . We denote this modification of LRR-IP model as BM-M2. The value 0.7 was found to be optimal in the explored range 0.5-0.8. It was found that in this way one could obtain a very pronounced improvement in the prediction of the flow field especially in the nonreacting case, with BM-M2 giving the correct decay rate of velocity on the symmetry axis, and also showing a very significant improvement for the rms fluctuation velocities, especially for the region downstream of the recirculation zone, as is illustrated in the next section.

\section{Results and Discussions}

The bluff-body burner investigated here is described in detail in [3]. The central fuel jet diameter is $3.6 \mathrm{~mm}$ and the diameter of the bluff-body is $50 \mathrm{~mm}$. Flow and mixing properties of both nonreacting and reacting flows have been measured. For the nonreacting case, air is used in the jet and the coflow and hence the flow has constant density. The bulk velocity of the jet is $61 \mathrm{~m} / \mathrm{s}$ and the coflow velocity is $20 \mathrm{~m} / \mathrm{s}$. For the reacting case, the central fuel jet $\left(\mathrm{CH}_{4} / \mathrm{H}_{2}=1 / 1\right.$, by volume) mixes and reacts with the coflow air stream. The bulk velocity of the jet is $108 \mathrm{~m} / \mathrm{s}$ and the coflow velocity is $35 \mathrm{~m} / \mathrm{s}$.

Here we present and discuss results of the numerical simulations of the nonreacting and the reacting flow using three DRSMs from the literature LRR-IP, JM and SSG (see Section 2) and two DRSMs obtained by changing a model constant in the LRR-IP model, denoted as BM-M1 and BM-M2 (see Section 4). The results are obtained using second-order upwind discretization.

\subsection{NONREACTING FLOW}

The computational results are presented as radial profiles at different axial locations; two available sets of experimental data [16] are also plotted for comparison. The two sets correspond to independent measurement campaigns and the 

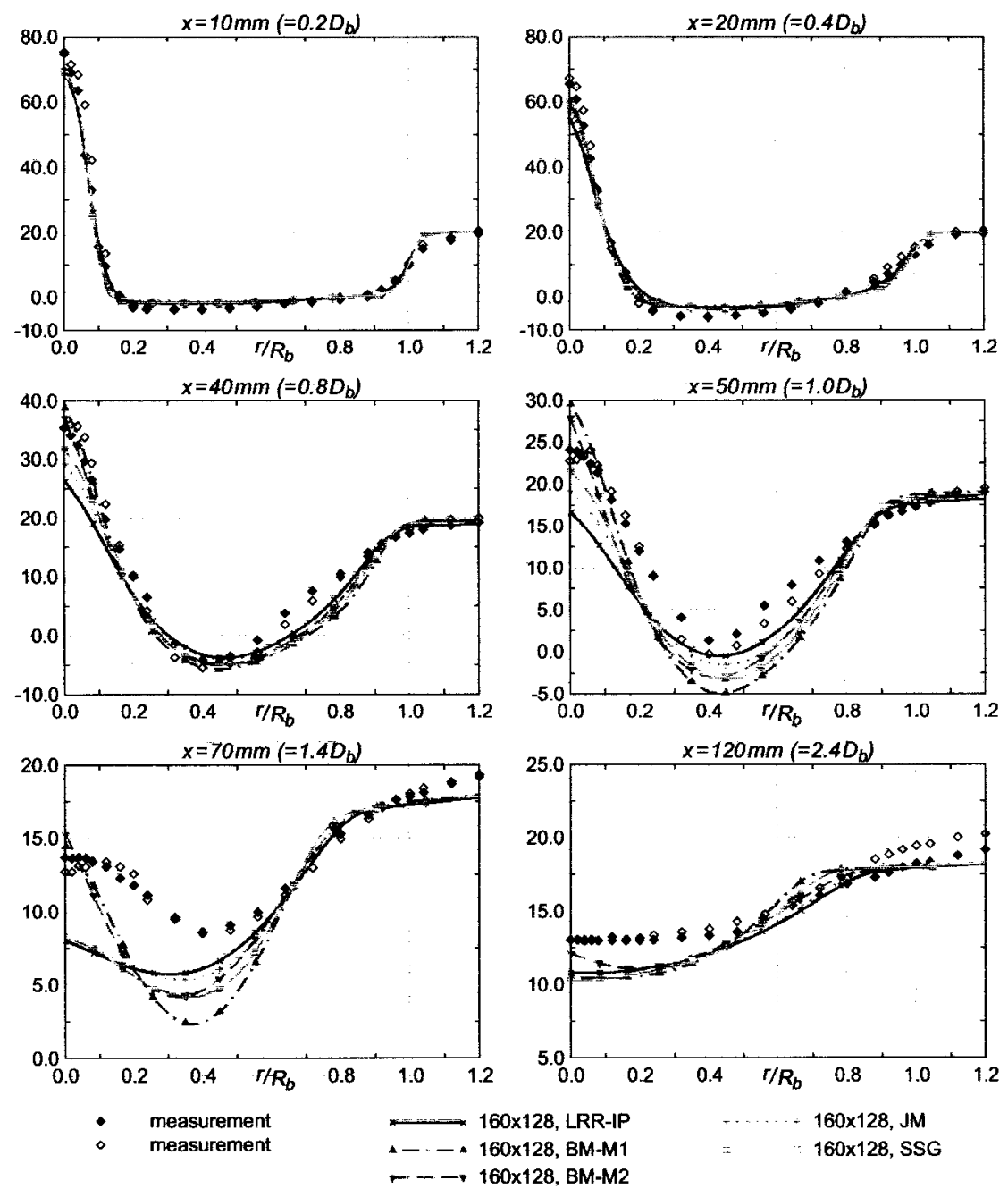

Figure 2. Radial profiles of mean axial velocity at different axial locations for the cold air flow around a bluff-body.

differences between both express the difficulty to exactly repeat an experiment for nominally the same boundary conditions.

Figure 2 shows the evolution of the mean axial velocity calculated with different DRSMs, the measured data are also plotted for the same locations as in the calculations.

A first criterion is the prediction of the recirculation zone length behind the bluff body. Experimentally, it is found to be $x / D_{b}=1.0$ [3], which is quite well predicted by the LRR-IP model $\left(C_{\epsilon 1}=1.44\right)$ and the JM model. The two modified LRR-IP models as well as the SSG model slightly overpredict the length of the recirculation zone. 
A second criterion is the prediction of the decay rate of axial velocity on the centreline. It can be clearly seen from the figure that the standard LRR-IP model considerably overpredicts the decay rate of velocity along the symmetry axis, and the discrepancy is larger further downstream. The SSG model gives better results than LRR-IP up to $x / D_{b}=1.0$, but further downstream deviates from the measured trends in the same way as LRR-IP. The result of the JM model is in between that of the LRR-IP model and that of the SSG model. In contrast, both of the modified LRR-IP models, i.e. BM-M1 and BM-M2, are showing agreement with the experimental data, also downstream of the recirculation zone at $x / D_{b}=1.4$, and therefore for this criterion do the best job. However the agreement away from the axis is less good.

Figure 3 shows the mean radial velocity component. Significant differences between the two measurement sets are seen at downstream distance $x / D_{b}=0.8$ and beyond. All models fail to correctly describe the evolution with axial distance of the two peaks in the radial profile. The peak near the centreline is underestimated by all the models. At the most downstream distance $x / D_{b}=2.4$ where the absolute value of radial velocity is lowest, the relative differences between the two measurement sets is largest. There the models tend to align with one of the two sets (open diamonds). Cylindrical symmetry, which is present by construction in the calculations implies vanishing mean radial velocity on the symmetry axis. All models have roughly the same performance, but the BM-M1 model deviates most from experiment.

Comparing Figures 2 and 3 it can be seen that the absolute value of the deviation between model prediction and experiment is smaller for mean radial velocity than for mean axial velocity.

Figure 4 shows the rms fluctuation of axial velocity. It is interesting to see that all the models show a reasonably good agreement with the experimental data up to $x / D_{b}=0.4$, and then the performance of the models begins to vary. On the whole the LRR-IP, JM and BM-M1 models deviate most from experiments. BM-M1 underpredicts fluctuations close to the centre line at $x / D_{b}=0.8$ and away from the centre line at $x / D_{b}=2.4$. LRR-IP and JM perform badly close to the centre line at $x / D_{b}=1.4$. There the SSG model and BM-M2 produce the better results. Far away from the axis at large downstream distance LRR-IP and JM are closest to the experimental data. This can partially be understood as a consequence of the quality of the predictions for the (gradients of) mean velocity that are felt in the rapid part of the pressure strain correlation. (SSG model: good predictions of mean velocity close to the centreline, LRR-IP model: good away from the centreline). Only BMM2 yields overall satisfactory results that show a reasonably good agreement with the experimental data, and also produces a good performance downstream of the recirculation zone. Nevertheless together with all other models it underestimates the level of rms axial velocity fluctuations at axial distance $x / D_{b}=2.4$ and radial distance larger than $r / R_{b}=0.8$. 

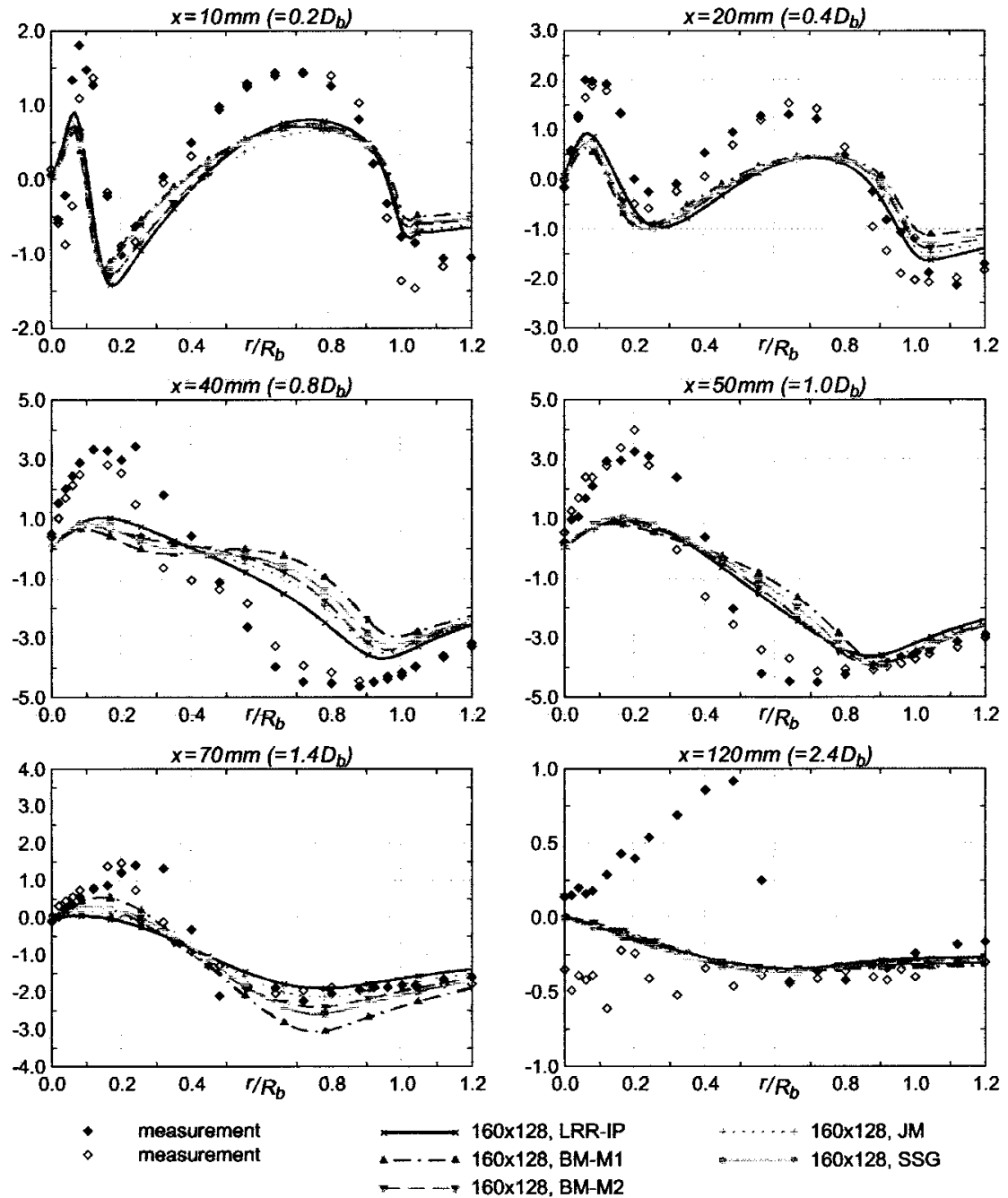

Figure 3. Radial profiles of mean radial velocity at different axial locations for the cold air flow around a bluff-body.

Figure 5 shows the rms fluctuation of the radial velocity, showing a very similar performance of the models as the rms fluctuating axial velocity. On the one hand, the predictions of the BM-M2 near the centreline for the rms fluctuation of velocities are as good as that of the BM-M1 model and better than that of the SSG model; on the other hand, the results in the region above the bluff-body are pretty well similar to the standard LRR-IP model and the JM model. In other words, it appears that BM-M2 combines the advantage of all models, yields the overall satisfactory results, and therefore keeps the full agreement with the experimental data along the whole axial direction, even further downstream. The complexity of the present flow at first is in the spatial structure of the mean flow and it seems that the extra term in 

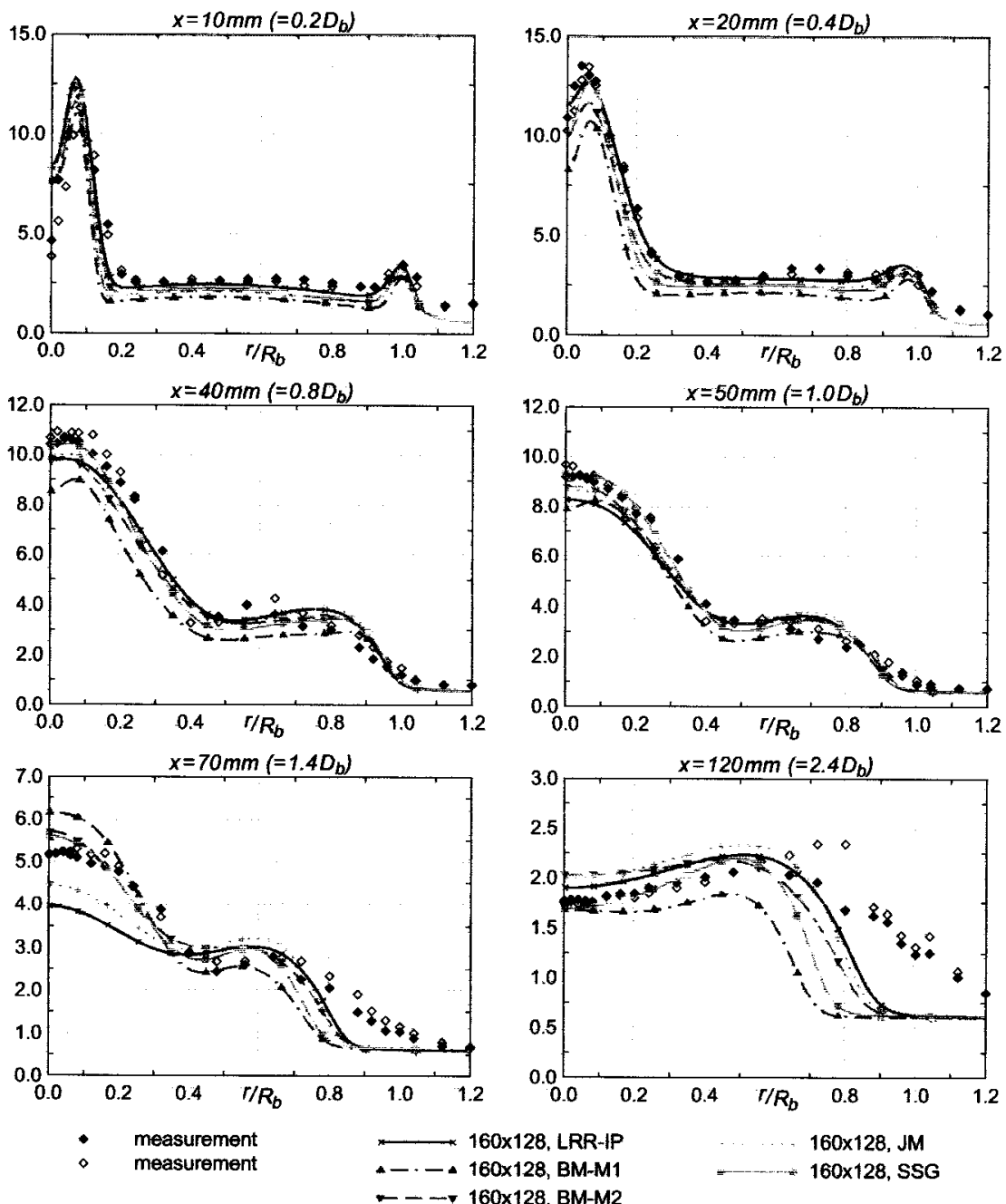

Figure 4. Radial profiles of rms fluctuation of axial velocity at different axial locations for the cold air flow around a bluff-body.

the slow part of the pressure strain term, which is nonlinear in the anisotropy tensor introduced in the SSG model, is not so adequate here. On the other hand the LRRIP model suffers from the consequences of the bad prediction of the decay rate of mean axial velocity. The first modification, acting on the dissipation equation, repairs this but deteriorates the predictions of mean flow away from the axis. On the other hand the second modification acts on the rapid part of the pressure strain term, and makes the response of the Reynolds-stress tensor to gradients of the mean field stronger. It appears then that this improves the mean fields away from the axis and at the same time maintains the centreline axial velocity decay rate reasonably close to experimental data. This is quite remarkable, indicating a quite significant 

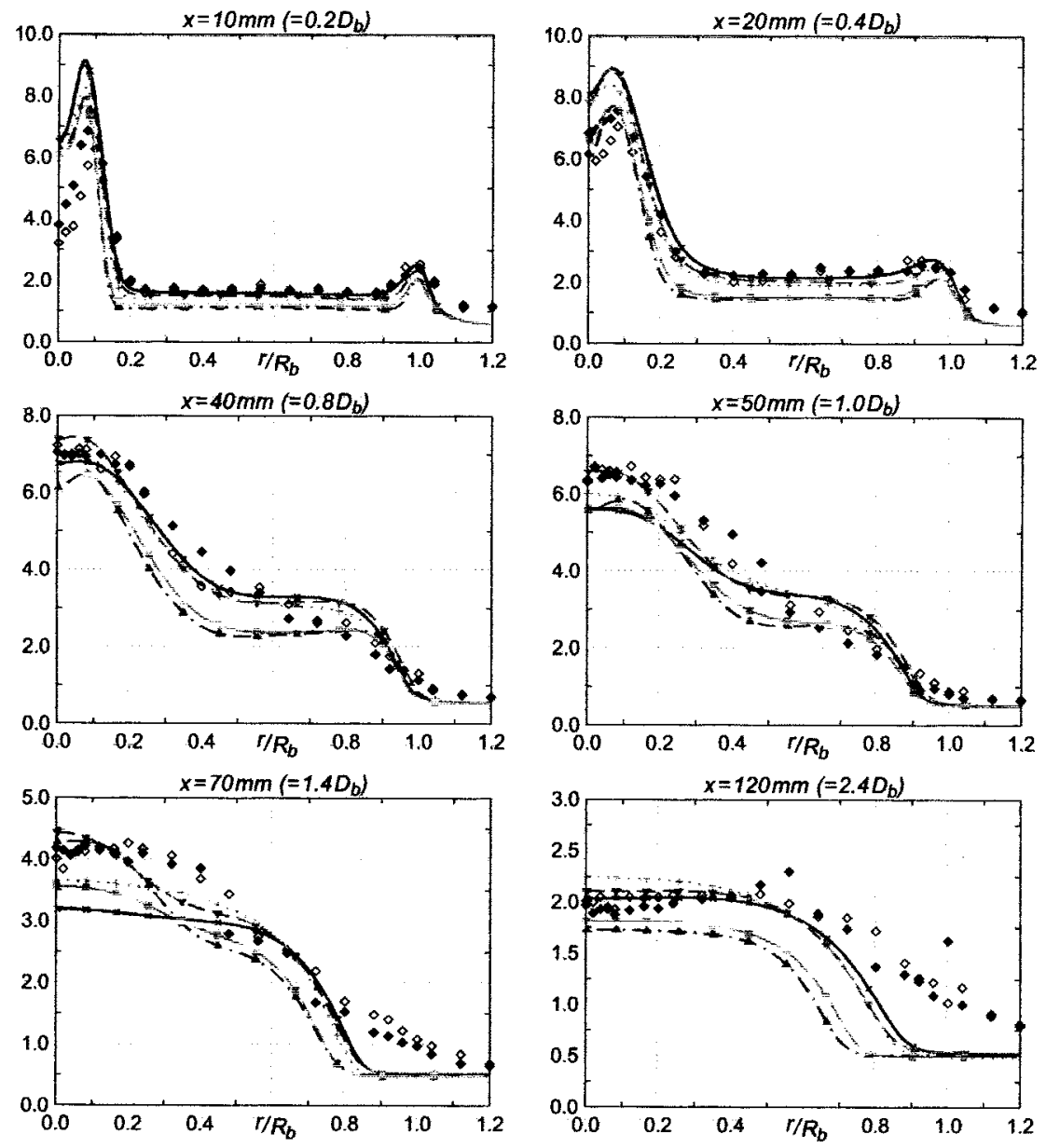

- measurement

$\longrightarrow 160 \times 128$, LRR-IP
$\rightarrow-\rightarrow 160 \times 128$, BM-M1

$\longrightarrow \rightarrow 160 \times 128, \mathrm{BM}-\mathrm{M} 2$

Figure 5. Radial profiles of rms fluctuation of radial velocity at different axial locations for the cold air flow around a bluff-body.

improvement compared to the results provided by the other models, considering that it is difficult to predict the rms fluctuation velocities correctly.

The predictions of turbulent shear stress are shown in Figure 6. Near the centreline in the recirculation zone, the SSG model as well as the two modified LRR-IP models can agree well with the experimental data, while the standard LRR-IP model and the JM model are less good. However in the region above the bluff-body, the standard LRR-IP model and the JM model yield results in close agreement with the experimental data even further downstream. But globally, only the BM-M2 is showing at least qualitatively overall good agreement with the experimental data. 

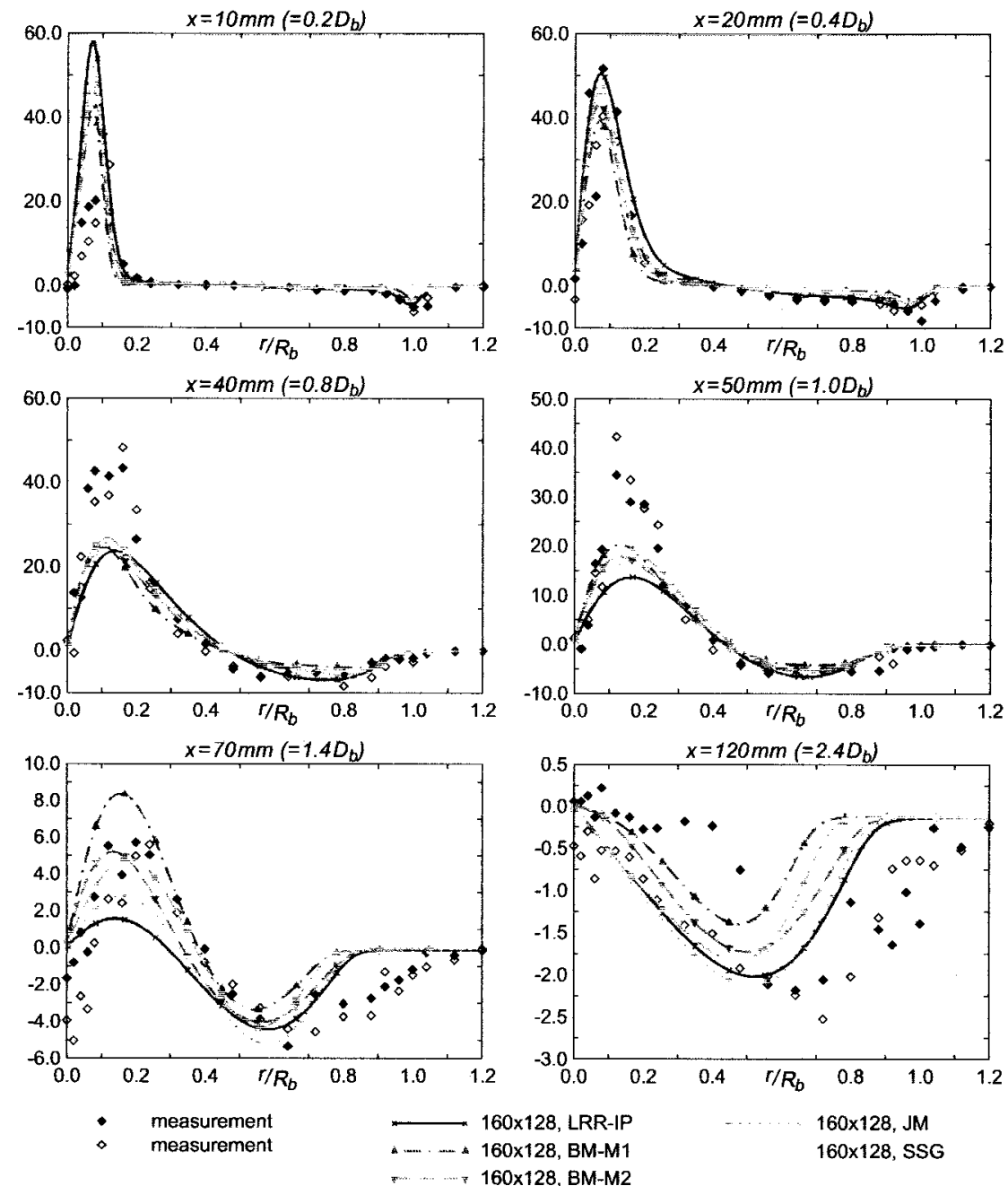

Figure 6. Radial profiles of turbulent shear stress at different axial locations for the cold air flow around a bluff-body.

We come to the following conclusion: good predictions of decay rate of axial velocity are only made by the BM-M1 and BM-M2 models. However, at the same time, the prediction of rms fluctuation velocities by BM-M1 are worse than those of the standard LRR-IP model and the JM model in the downstream region after the round jet has been severely influenced by the nonjet like end of the recirculation zone. In other words, changing $C_{\epsilon 1}$ from 1.44 to 1.6 is not an optimal way to improve the overall prediction. On the other hand in the BM-M2, the model constant $C_{\epsilon 1}$ is kept unchanged, the model constant $C_{2}$ in the pressure strain model is changed from 0.6 to 0.7 , making the effect of gradients of mean velocity on the evolution of Reynolds stresses stronger while not changing the strength of the 
slow return to isotropy part. In this way the decay rate of the axial velocity is correctly estimated similar to the BM-M1 prediction, meanwhile the prediction of rms fluctuation of velocities is getting better than that of BM-M1.

\subsection{REACTING FLOW}

For the reacting flow, the central jet consisting of a mixture of $\mathrm{CH}_{4} / \mathrm{H}_{2}(1: 1$ by volume) mixes with the coflow air, and the flame is stabilised behind the bluff-body by the intense mixing of fuel and air and by the hot products providing a continuous ignition source in the recirculation zone [3]. In the simulation, a full equilibrium model has been chosen as chemistry model and a beta function shape for the PDF of mixture fraction is assumed. In addition to the equations of the Reynolds-stress model, transport equations are solved for (Favre) mean and variance and Reynolds flux of mixture fraction. Here the computation results for the flow field as well as the mixing field are compared with the experimental data.

As in the previous section we present radial profiles of variables at different axial locations calculated with three standard DRSMs (LRR-IP, JM and SSG) and two modified forms of LRR-IP (BM-M1 and BM-M2). Also plotted are the experimental data (two measurement sets, except at $x / D_{b}=2.4$, where only one measurement set is available for the flow field). It is important to remark that the level of agreement between measurements and predictions in the reacting case is influenced by both turbulence model and chemistry model and therefore the conclusions on performance of turbulence models strictly speaking are valid only when the mean density field is predicted correctly. In the equilibrium chemistry approach used here the density is determined by mixture fraction. In general, finite rate chemistry may have to be included to have a correct representation of the density and to arrive at a final conclusion on the performance of turbulence models. This may be the subject of a follow study to this work.

\subsubsection{Flow Field}

Figure 7 shows the radial profiles of axial velocity. In the reacting case, the length of recirculation observed in the experiment is longer than in the nonreacting case, namely 1.6 bluff-body diameters [3], which is well predicted by the BM-M1 model (the BM-M1 model still has a small region with negative mean axial velocity at $\left.x / D_{b}=1.4\right)$. All other models underestimate the length of recirculation zone (showing no region with negative axial velocity at $x / D_{b}=1.4$ ).

The decay rate of axial velocity is well predicted by BM-M1 and especially BM-M2 up to $x / D_{b}=1.0$. SSG, JM and LRR-IP overpredict the decay rate as found in the nonreacting case. For small axial distances the differences between the model predictions away from the axis are small. Greater differences appear at $x / D_{b}=1.4$ and $x / D_{b}=2.4$. Looking at the radial profiles as a whole, BM-M1 is showing best agreement with the experimental data at $x / D_{b}=1.4$, while at $x / D_{b}=2.4$ BM-M1 fails and BM-M2 has the better performance. This illustrates 

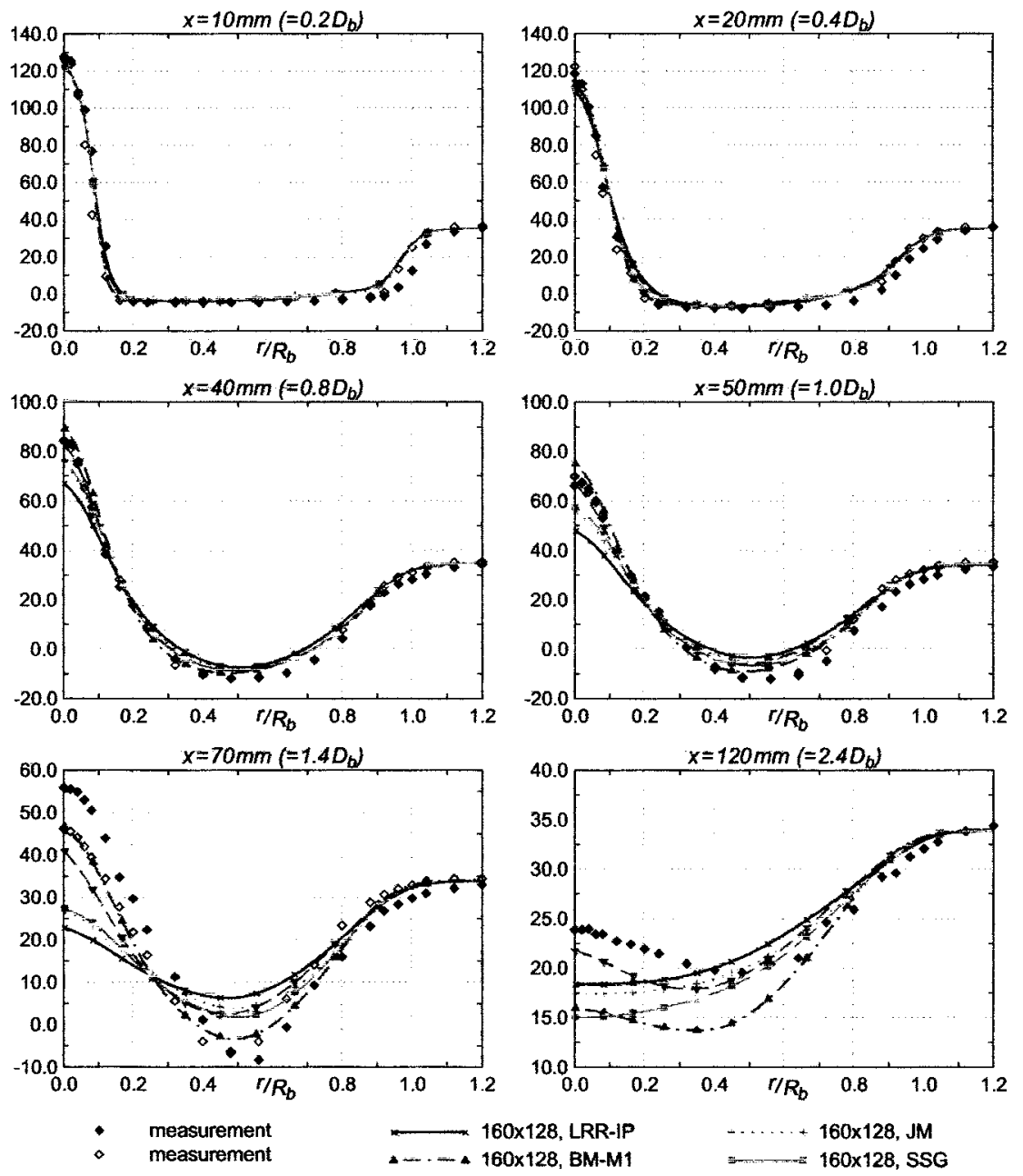

Figure 7. Radial profiles of mean axial velocity at different axial locations for the reacting flow stabilised on a bluff-body.

the difficulty to obtain consistent good predictions in the region downstream of the recirculation zone.

The profiles of mean radial velocity are shown in Figure 8. Compared to the nonreacting case, there is a smaller difference between the two measurement sets and the predictions are in better agreement with experiment up to $x / D_{b}=0.4$. At axial distance $x / D_{b}=0.8$ the LRR-IP models performs best up to radial distance $r / R_{b}=0.4$ and the BM-M1 model performs best at larger radial distance, with SSG being second best. At this axial distance the measurements show a significant qualitative difference between the nonreacting and the reacting case between $r / R_{b}=0.4$ and $r / R_{b}=0.8$ which is not reproduced by the models. The 

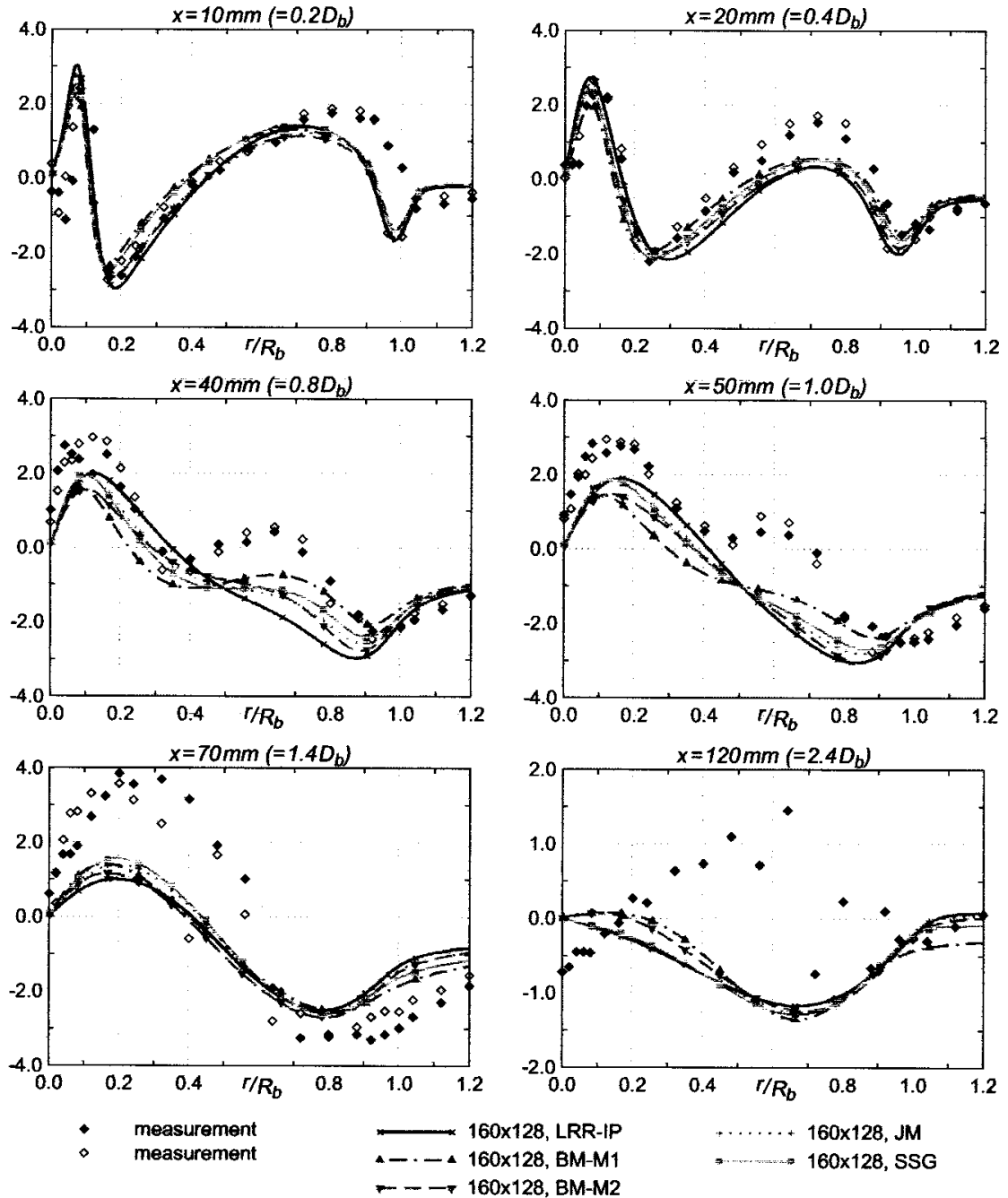

Figure 8. Radial profiles of mean radial velocity at different axial locations for the reacting flow stabilised on a bluff-body.

discrepancy between the calculations and measurements further downstream, may be partly attributed to the inaccuracies in the radial component measurements as discussed in [3].

Figure 9 shows the radial profiles of rms fluctuation of the axial velocity. Except for BM-M1 the models slightly overpredict the fluctuations up to $x / D_{b}=0.8$. On the other hand at $x / D_{b}=1.4$ all models underpredict the fluctuations. LRR-IP gives the largest deviation with experiment there. At $x / D_{b}=2.4$, downstream of the recirculation zone, the BM-M1 and BM-M2 models show significantly better agreement with experiment than LRR-IP, SSG and JM. Figure 10 shows the radial profiles of rms fluctuation of the radial velocity. The models perform in a similar 

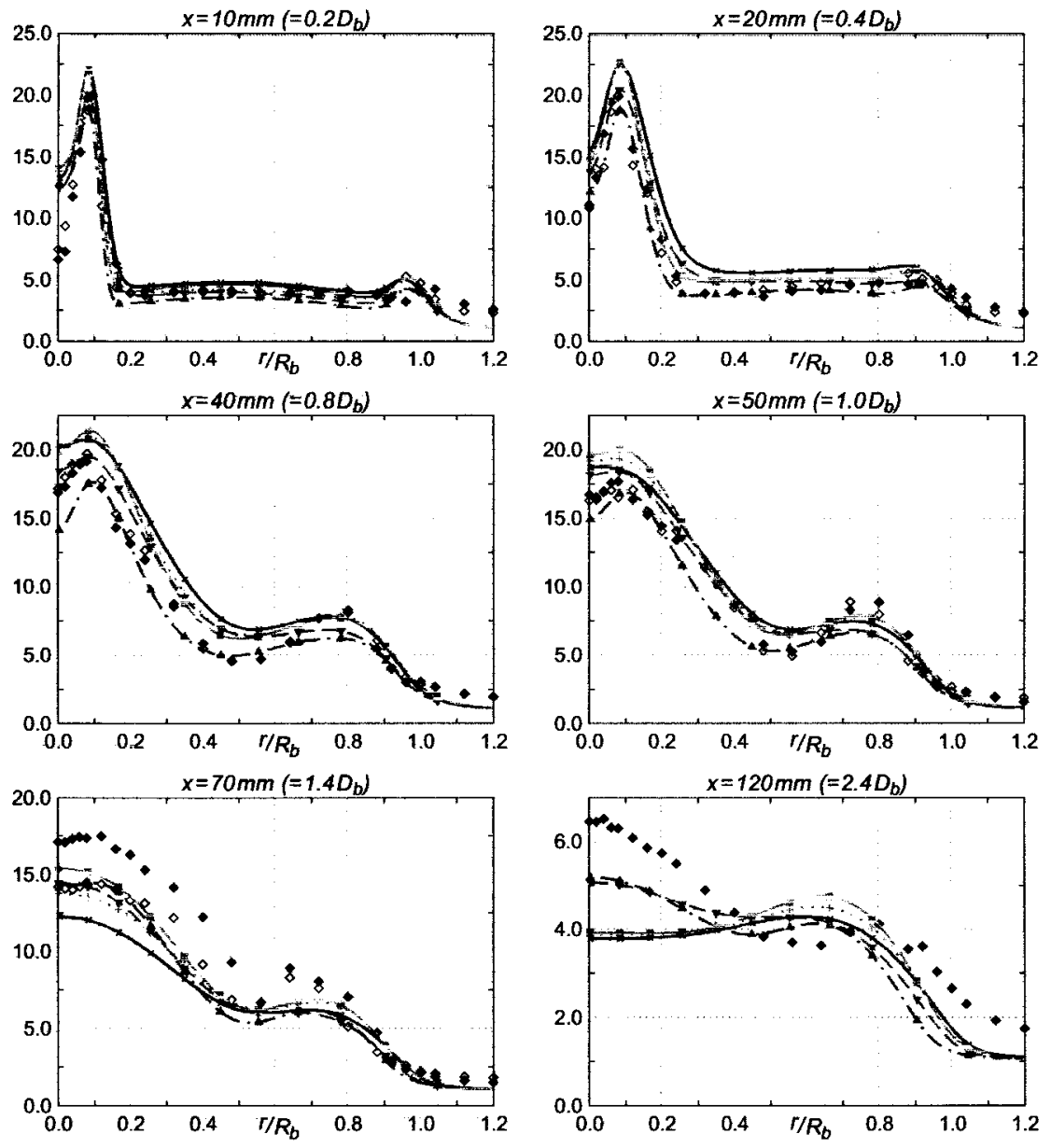

- measurement

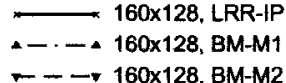

$160 \times 128, \mathrm{JM}$

measurement $\quad \ldots 160 \times 128, B M-M 1$

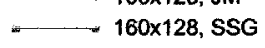

Figure 9. Radial profiles of rms fluctuation of axial velocity at different axial locations for the reacting flow stabilised on a bluff-body.

way as for the axial velocity fluctuations. Up to $x / D_{b}=0.8, \mathrm{BM}-\mathrm{M} 1$ gives the best prediction, with SSG as second best. In the region downstream of the recirculation zone all models underpredict the radial fluctuations. The relatively best agreement with experiment is shown by BM-M2.

For the mean turbulent shear stress, it is difficult to make any solid conclusions because there is a large error margin in the measured data (up to $30 \%$ error) near the axis [16]. However Figure 11 indicates that up to $x / D_{b}=1.0$ the differences between the models are relatively small, and up to $x / D_{b}=0.8$ the peak in the radial profile is overpredicted. On the whole BM-M1 shows best agreement with the measurements, with BM-M2 second best. 

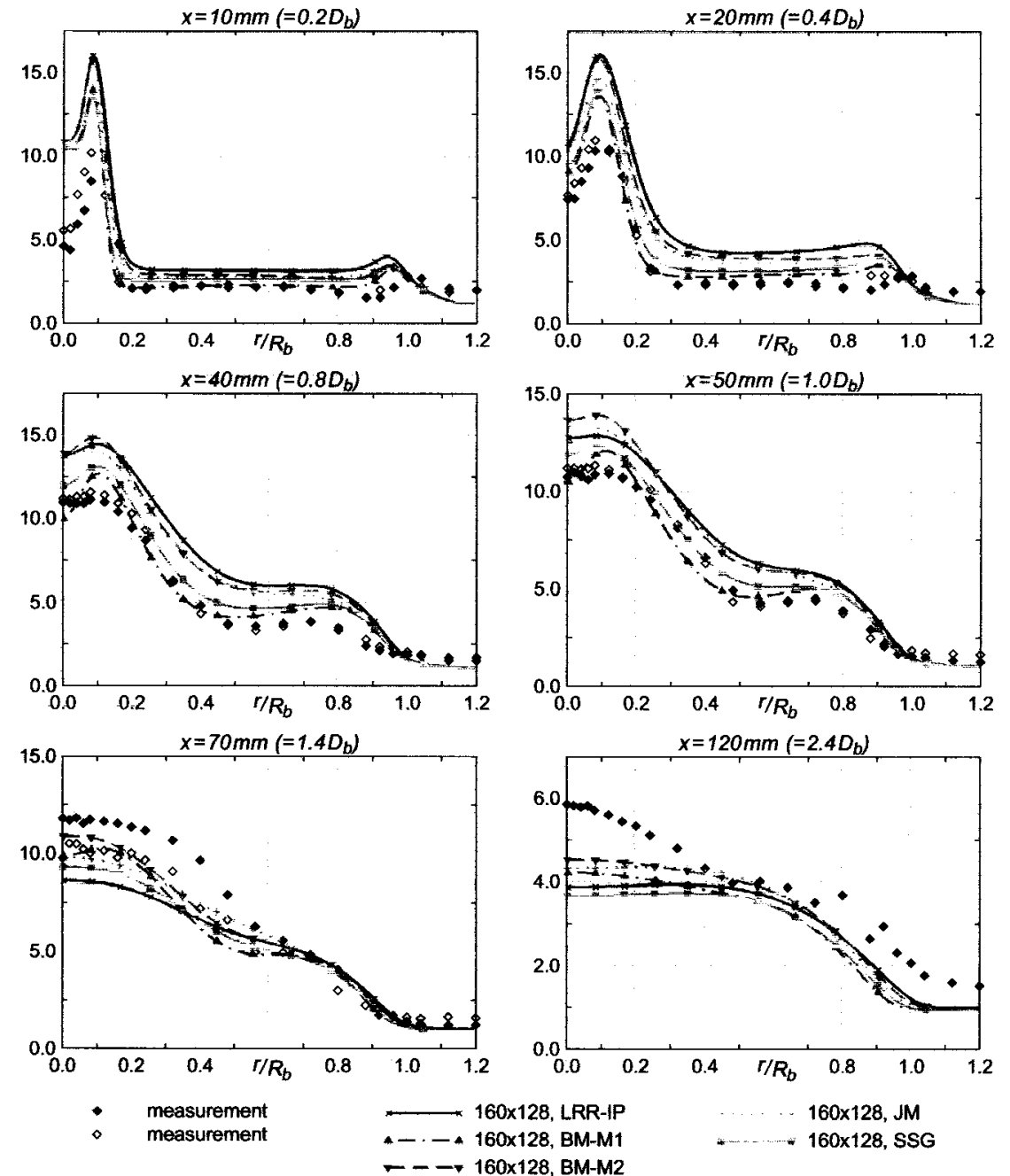

Figure 10. Radial profiles of rms fluctuation of radial velocity at different axial locations for the reacting flow stabilised on a bluff-body.

We come to the following conclusion: the best predictions for the mean and fluctuating properties of velocity are provided by the BM-M1 and BM-M2 models. The predictions of SSG, JM and standard LRR-IP are less good and of similar quality. Both BM-M1 and BM-M2 give good agreement for the decay rate of axial velocity up to $x / D_{b}=1.4$. Further downstream only BM-M2 gives a good prediction.

The better performance of BM-M2 compared to BM-M1 observed for the nonreacting flow is not so pronounced in the reacting flow. This illustrates that the relative performance of the different DRSMs may vary due to presence of reaction and variable density. The reasons for this are not fully understood. Due to expan- 

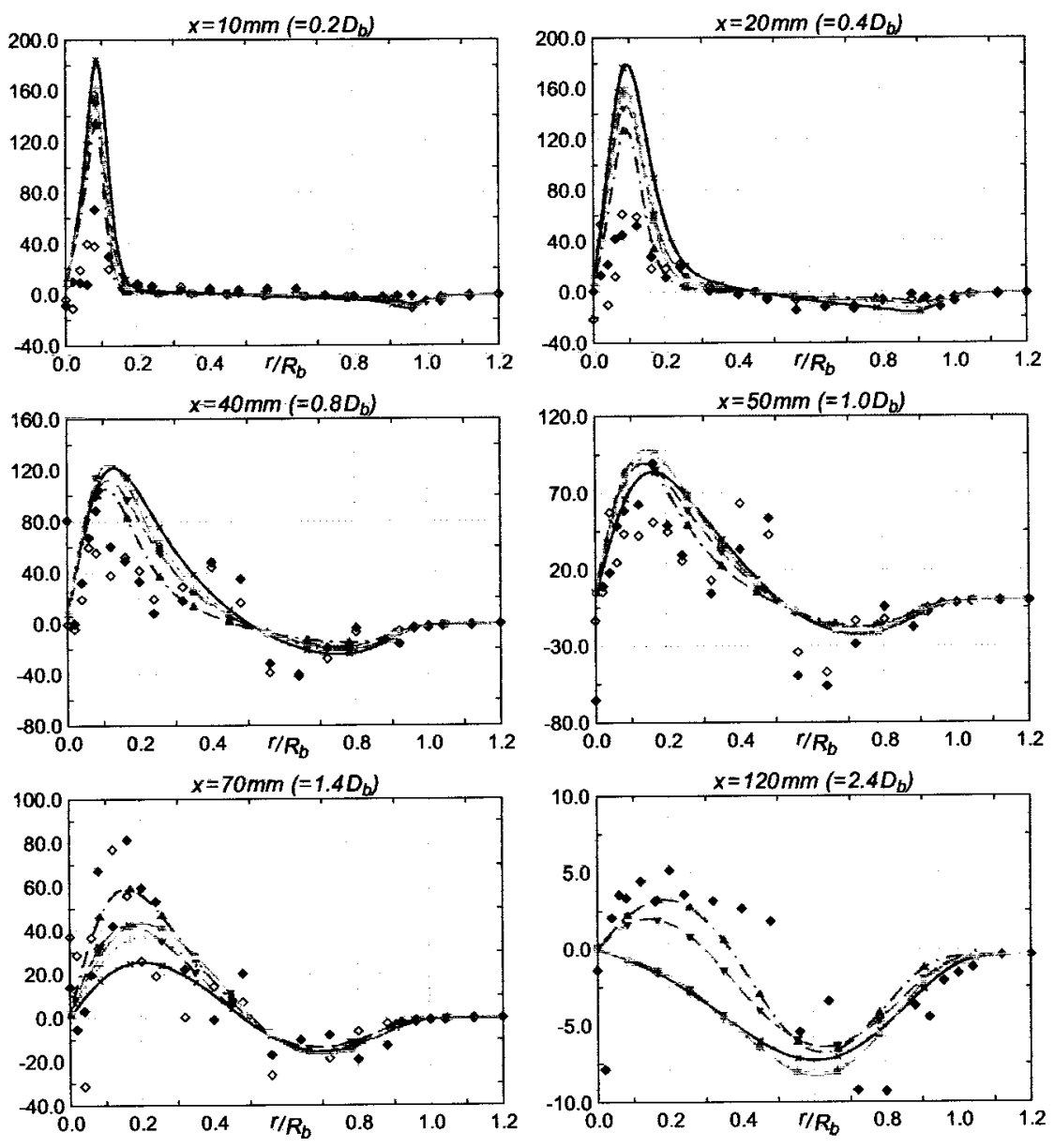

- measurement

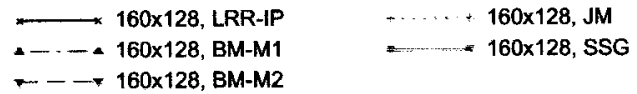

Figure 11. Radial profiles of turbulent shear stress at different axial locations for the reacting flow stabilised on a bluff-body.

sion effects there is a difference in length of the recirculation region. It seems then that due to these effects the relative impact of changes in the model constant in the dissipation rate equation and in the rapid part of the pressure strain term is different. This may touch upon the general question of adequacy of turbulence modelling of variable density flow using Favre averaging.

\subsubsection{Mixing Field}

Here the simulation results for mean and standard deviation of mixture fraction and for mean temperature are presented and compared to measurement data. It should be remarked that no measurement data are available for the inflow conditions at 

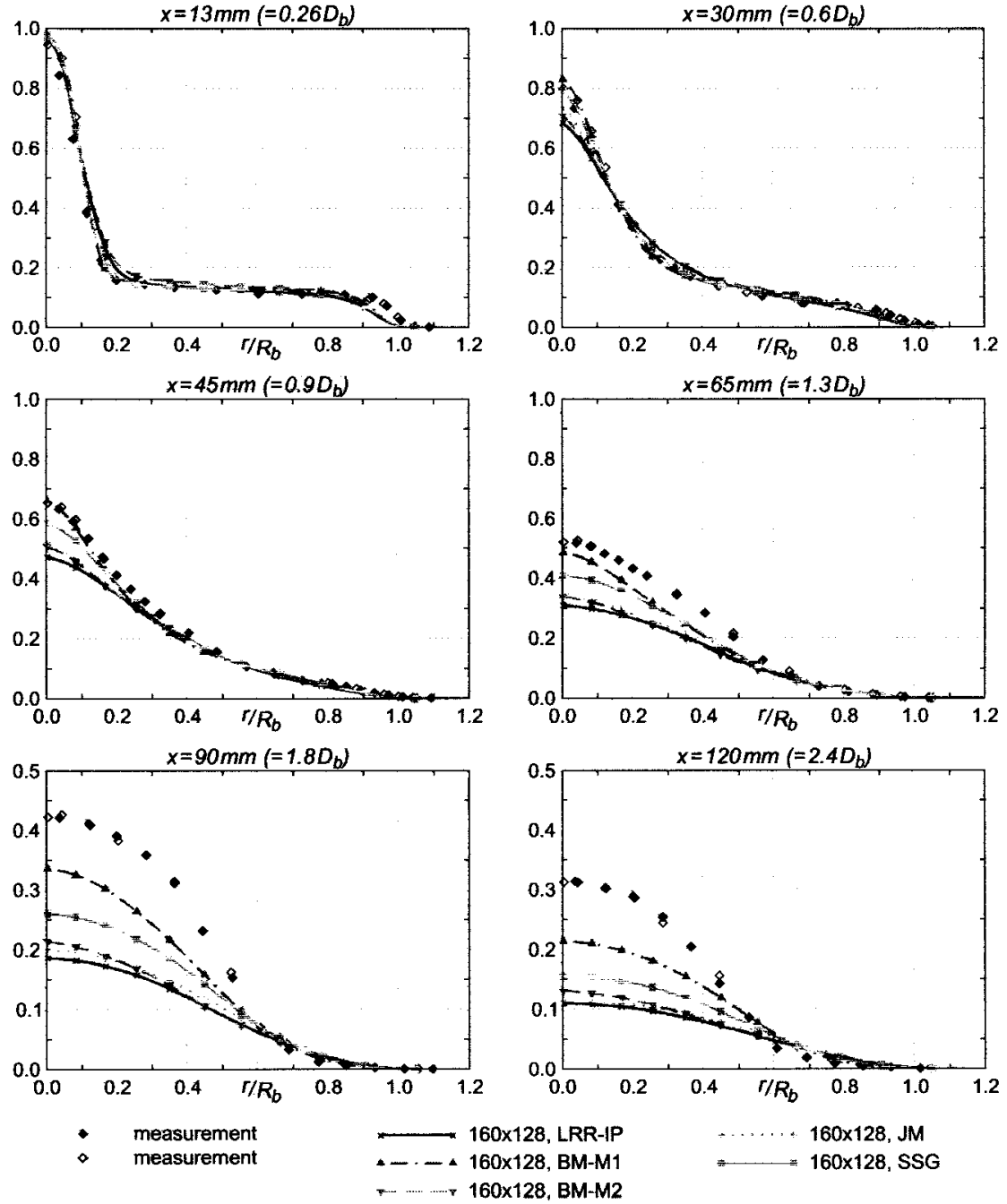

Figure 12. Radial profiles of mean mixture fraction at different axial locations for the reacting flow stabilised on a bluff-body.

which the velocity was measured, but rather for the case in which the jet and coflow bulk velocities were 118 and $40 \mathrm{~m} / \mathrm{s}$, respectively, instead of 108 and $35 \mathrm{~m} / \mathrm{s}$ [16]. It has been checked that for the profiles considered here this is of minor importance. The conclusions on the mixing fields are independent of this difference.

Figure 12 shows profiles of mean mixture fraction. One would expect that the decay rate of mean axial velocity on the symmetry axis is coupled to the decay rate of mean mixture fraction and that therefore the quality of the prediction for both quantities by a certain model would be the same. This is found not to be the case. The decay rate of mean mixture fraction is overpredicted by all models and the best prediction comes from the BM-M1 model, with SSG as second best. 

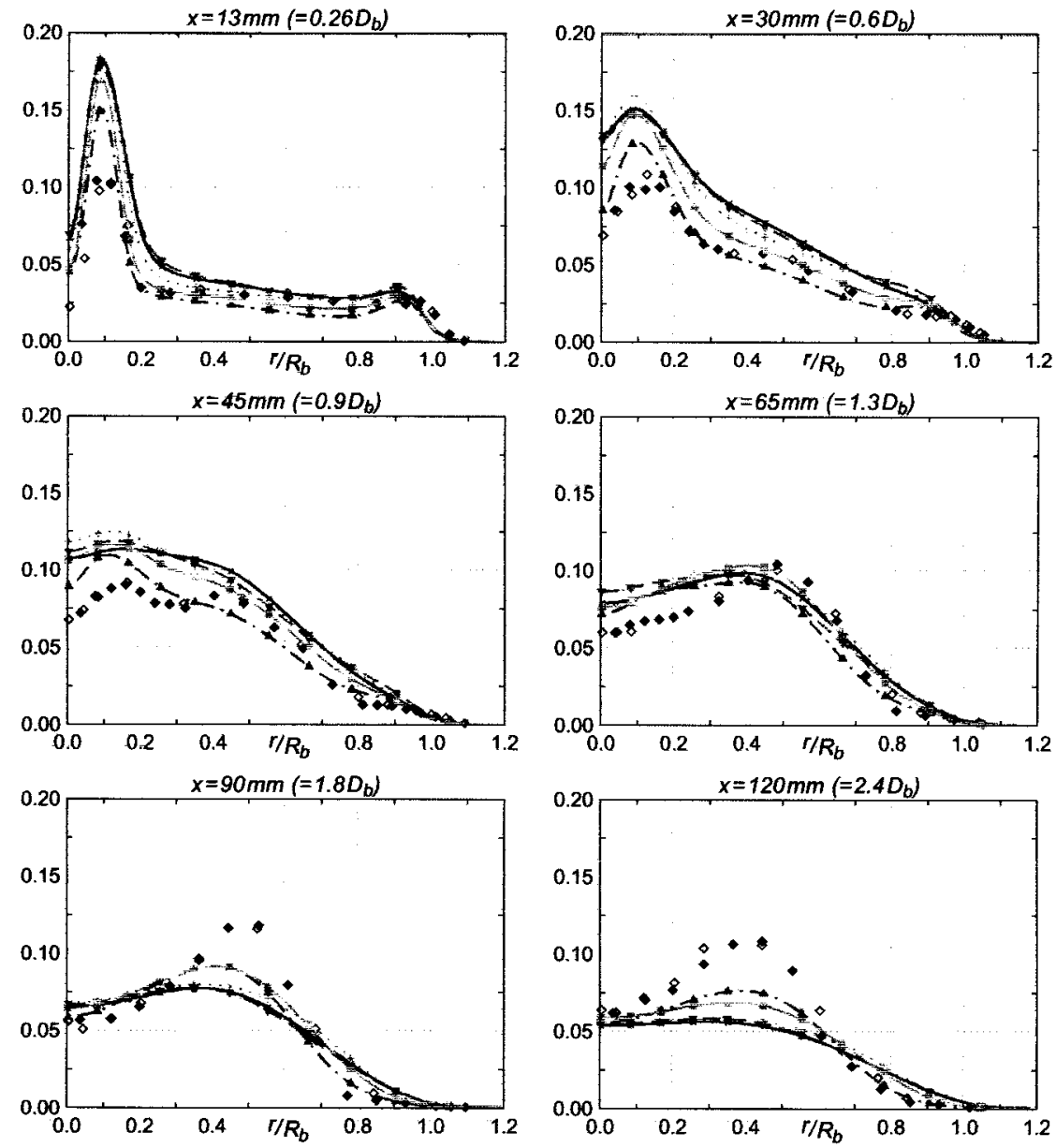

measurement

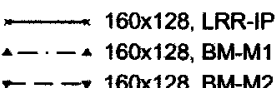

$160 \times 128, \mathrm{JM}$ $\multimap \rightarrow 160 \times 128, B M-M 2$

Figure 13. Radial profiles of rms fluctuation of mixture fraction at different axial locations for the reacting flow stabilised on a bluff-body.

This result can be understood from the fact that in both cases (BM-M1 and SSG) a model constant in the dissipation equation has been changed, which directly affects the centreline decay rate. On the other hand, the result of BM-M2 (good prediction of mean velocity centre line decay rate but bad prediction of mean mixture fraction centre line decay rate) shows that simply changing a model constant in the pressure strain term does not guarantee this coupling effect. Maybe this also illustrates then why an optimal form of pressure strain term as looked for in the SSG model had to be combined with a change in model constant in the dissipation equation [27]. 

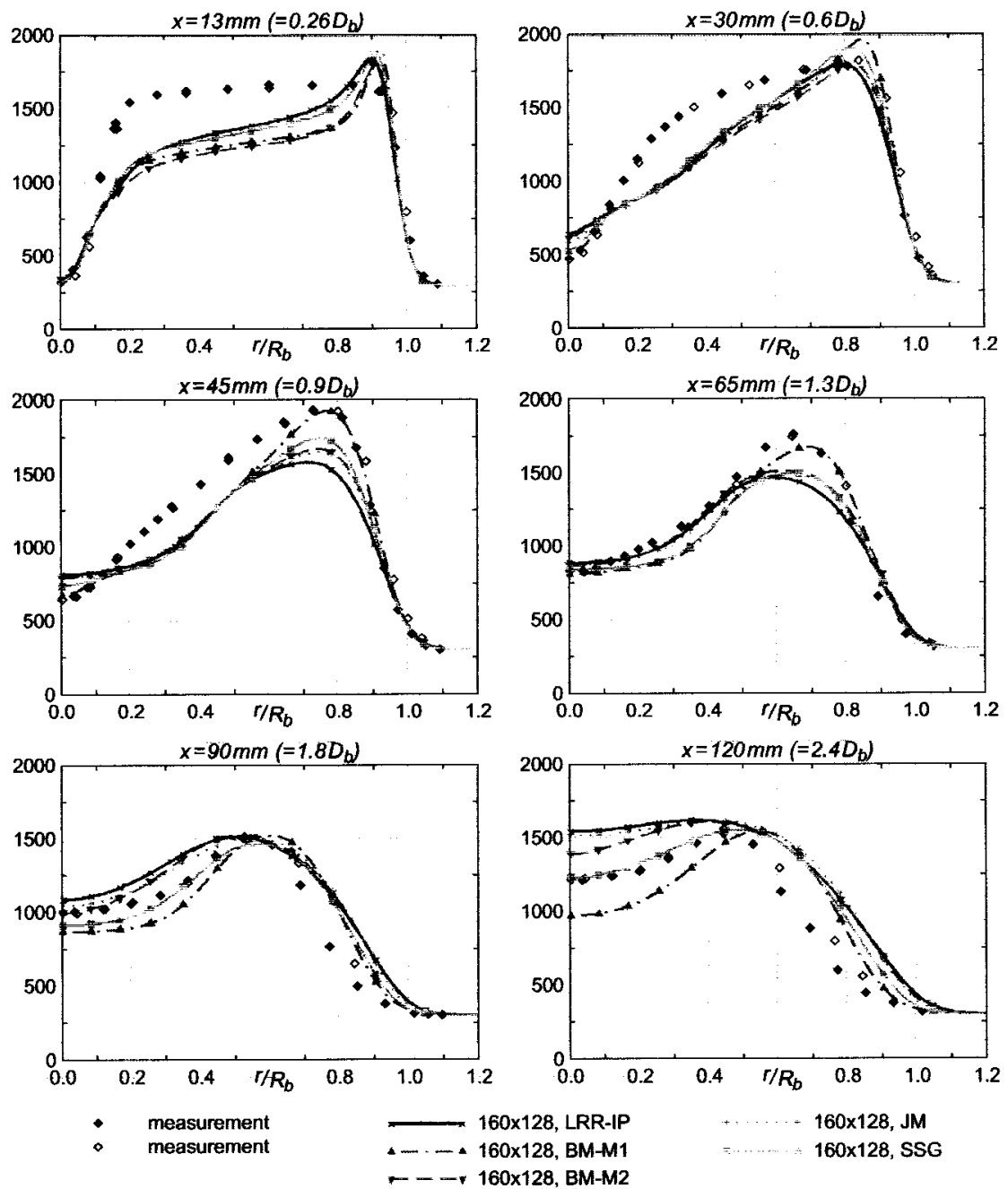

Figure 14. Radial profiles of mean temperature at different axial locations for the reacting flow stabilised on a bluff-body.

Figure 13 shows the rms fluctuation of mixture fraction plotted at different axial locations in the same flame. Again it is apparent that the BM-M1 and SSG models yield prediction in closest agreement with the experimental data.

Figure 14 shows the radial profiles of the mean temperature at different axial locations. In the framework of the fast chemistry model temperature is function of mixture fraction and most relevant results are those of models that predict the mean and rms fluctuation of mixture fraction correctly, the BM-M1 and SSG models. Therefore the fact that in the near field, at locations $x / D_{b}=0.26$ and 0.6 , the standard LRR-IP model provides better results than the BM-M1 and SSG model must be considered an effect of cancellation of errors. At the rich side of stoichio- 
metric mixture fraction, (near the fuel jet) the equilibrium assumption leads to a systematic underprediction of temperature. In the shear layer (at the outer edge of the bluff body) the fast chemistry approach systematically overpredicts temperature because local extinction is ruled out. Further downstream, the adverse effects of using equilibrium chemistry are less severe and predicting properties of mixture fraction correctly is already sufficient to reach a good prediction of temperature. Accordingly at $x / D_{b}=0.9$ and $x / D_{b}=1.3$ the BM-M1 predictions come closest to the experimental data. Further downstream, where the predictions for the mixing field are getting worse, the relatively best agreement is found for SSG but this may also be caused by cancellation of the effects of errors in the mean and the variance of mixture fraction.

\section{Conclusions}

A bluff-body stabilised flame and the associated nonreacting flow have been numerically investigated with several differential Reynolds-stress models. The mixing (and reaction) is described by a simple mixture fraction based model. Transport equations for mean mixture fraction, variance of mixture fraction and Reynolds flux of mixture fraction are solved.

Grid independence of the results was demonstrated. In relation to grid refinement we have observed that it is far more important to put enough number of cells (in the radial direction) located in the bluff-body region to obtain the gridindependent solutions, while the number of cells in the jet and coflow have a little effect on the grid independence.

In order to clarify the applicability of the various models, three typical DRSMs from the literature (LRR-IP, JM, SSG) and two modified versions of the basic LRR-IP model (called BM-M1 and BM-M2) have been applied. In both modified versions only one of the model constants is changed from its standard value. In BM-M1 the model constant $C_{\epsilon 1}$ is modified from 1.44 to 1.6 , as proposed earlier by Dally et al. [3] for this flame, in BM-M2 the model constant $C_{2}$ in the rapid part of the LRR-IP pressure strain model is modified from 0.6 to 0.7 .

We have observed that on the grids used, the detailed results for the nonreacting case depend on the order of the discretization scheme (first order upwind or second order upwind). The difference between the results using a different scheme can be as large as the differences between the models. Nevertheless, the overall conclusions on the relative performance of the models were found to be independent of the scheme. For the reacting case the dependence on the order of the discretization scheme was found to be smaller than the differences between the models and almost negligible. The results presented and discussed above all have been obtained using the second order scheme.

It is found that the ranking of the performance of the considered Reynolds-stress models is different in the nonreacting flow and the reacting flow and therefore we have to differentiate our conclusions for the two cases. 
In both the nonreacting and the reacting case it is found that by modifying a single model constant in the standard LRR-IP model, better predictions can be obtained than by any of the three standard DRSMs considered (LRR-IP, SSG and $\mathrm{JM})$. It is confirmed that a simple modification to the $C_{\epsilon 1}$ constant $\left(C_{\epsilon 1}=1.6\right)$ in the dissipation equation together with LRR-IP model (BM-M1 model) can give better results for axial velocity as well as radial velocity. In the nonreacting case this does not lead to improvement for rms fluctuating velocity prediction especially further downstream. However in the reacting case it does also lead to improvement in rms fluctuation velocity prediction.

Motivated by the need to improve the prediction results, a new modification of the LRR-IP model (i.e. BM-M2 model) has been proposed which can provide overall better predictions compared to the other DRSMs, not only for the axial velocity, but also for the rms fluctuating velocities. With BM-M2 a significant improvement of the prediction of the flow field is obtained in the nonreacting case. The BM-M2 model combines a good prediction of the centre line mean velocity profile with good predictions of the fluctuations on and away from the axis. The pronounced improvement in the quality of predictions of rms velocity fluctuations in the downstream region is remarkable. Hence, for the prediction of the flow field in the nonreacting case the BM-M2 model comes out as best choice.

For the prediction of the flow field in the reacting case both BM-M1 and BMM2 perform well. However, for the prediction of the mean mixture fraction BM-M2 is not adequate and BM-M1 performs best with SSG as second best. Taking into account the quality of predictions for both flow field and mixture fraction, we conclude that on the basis of the present study which has used the simple equilibrium chemistry assumed shape PDF model, the BM-M1 model is the recommended choice of DRSM for simulations of the bluff-body flame. Among the three unmodified DRSMs considered (LRR-IP, SSG and JM), SSG gives relatively the best results.

Further progress in predicting mean temperature and species concentrations can now be made by using a more sophisticated chemistry model and turbulencechemistry interaction model (e.g. flamelet model, Monte Carlo PDF model, CMC) in combination with these relatively most successful DRSMs. Because of the role of mean density in the equations of the turbulence model, the changes in the chemistry model may have impact on the performance of the turbulence model and it will remain necessary to check also the quality of the predictions of mean and rms velocity. First results of Monte Carlo simulations in combination with BM-M1 are reported in [21].

\section{Acknowledgements}

In the initial phase of this work B. Naud was Ph.D. student at the Thermal and Fluids Sciences Section, TU Delft, supported by the Foundation for Fundamental 
Research on Matter (FOM) and Guoxiu Li was having a sabbatical leave at TU Delft, supported by a research fellowship from Delft University of Technology.

\section{References}

1. Craft, T.J. and Launder, B.E., Computation of impinging flows using second-moment closures. In: Proceedings of the 8th Symposium on Turbulent Shear Flows, TU Munich, Germany (1991) pp. 851-856.

2. Daly, B.J. and Harlow, F.H., Transport equations in turbulence. Phys. Fluids 13 (1970) 26342649.

3. Dally, B.B. and Masri, A.R., Flow and mixing fields of turbulent bluff-body jets and flames. Combust. Theory Modeling 2 (1998) 193-219.

4. Dally, B.B., Masri, A.R., Barlow, R.S. and Fiechtner, G.J., Instantaneous and mean compositional structure of bluff-body stabilized nonpremixed flame. Combust. Flame 114 (1998) 119-148.

5. Fu, S., Launder, B.E. and Tselepidakis, D.P., Accommodating the effects of high strain rates in modeling the pressure-strain correlation. Mechanical Engineering Department Report TFD/87/5, University of Manchester Institute of Science and Technology (1987).

6. Hanjalic, K. and Launder, B.E., A Reynolds stress model of turbulence and its application to thin shear flows. J. Fluid Mech. 52 (1972) 609-638.

7. Hanjalic, K. and Jakirlic, S., Second-moment turbulence closure modelling. In: Launder, B. and Sandham, N. (eds.), Closure Strategies for Turbulent and Transitional Flows. Cambridge University Press, Cambridge (2002) pp. 47-101.

8. Hossain, M., Jones, J.C. and Malalasekera, W., Modelling of a bluff-body nonpremixed flame using a coupled radiation/flamelet combustion model. Flow, Turbulence and Combustion 67 (2001) 217-234.

9. Jenny, P., Pope, S.B., Muradoglu, M. and Caughey, D.A., PDF simulations of a bluff-body stabilized flow. J. Comput. Phys. 169 (2001) 1-23.

10. Jones, W.P. and Musonge, P., Closure of the Reynolds stress and scalar flux equations. Phys. Fluids 31 (1988) 3589-3604.

11. Jones, W.P., Turbulence modelling and numerical solution methods for variable density and combusting flows. In: Libby, P. and Williams, F. (eds.), Turbulent Reacting Flows. Academic Press, London (1994) pp. 309-374.

12. Kim, S.H., Huh, K.Y. and Tao, L., Application of the elliptic conditional moment closure model to a two-dimensional nonpremixed methanol bluff-body flame. Combust. Flame 120 (2000) 75-90.

13. Kim, S.H. and Huh, K.Y., Use of the conditional moment closure model to predict NO formation in a turbulent CH4/H2 flame over a bluff-body. Combust. Flame 130 (2002) 94-111.

14. Launder, B.E., Reece, G.J. and Rodi, W., Progress in the development of a Reynolds-stress turbulence closure. J. Fluid Mech. 68 (1975) 537-566.

15. Li, G., Naud, B. and Roekaerts, D., The effect of grid and turbulence model on the simulation of a bluff-body flame with Reynolds-stress model. In: Barlow, R.S. and Pitsch, H. (eds.), Proceedings of the 6th International Workshop on Measurement and Computation of Turbulent Nonpremixed Flames, Japan. http://ww.ca.sandia.gov/TNF (2002) pp. 65-66.

16. Masri, A. R., http://www.mech.eng.usyd.edu.au/thermofluids/tnf6/tnf6bb.htm, The University of Sydney, dataset HM1E.

17. Masri, A.R., Computation of bluff-body stabilized jets and flames. In: Proceedings of 5th International Workshop on Measurement and Computation of Turbulent Nonpremixed Flames, Delft, The Netherlands. http://ww.ca.sandia.gov/TNF (2000) section 3. 
18. McGuirk, J.J. and Rodi, W., The calculation of three dimensional turbulent free jets. In: Durst, F., Launder, B.E., Schmit, F.W. and Whitelaw, J.H. (eds.), Proceedings of the 1st Symposium on Turbulent Shear Flows. Springer-Verlag, Berlin (1979) pp. 71-83.

19. Merci, B., Dick, E., Vierendeels, J., Roekaerts, D. and Peeters, T.W.J., Application of a new cubic turbulence model to piloted and bluff-body diffusion flames. Combust. Flame 126 (2001) 1533-1556.

20. Muradoglu, M., Liu, K. and Pope, S.B., PDF modeling of a bluff-body stabilized turbulent flame. Combust. Flame 132 (2003) 115-137.

21. Naud, B., PDF modeling of turbulent sprays and flames using a particle stochastic approach. Ph.D. Thesis, Delft University of Technology (2003).

22. Peeters, T.W.J., Numerical modeling of turbulent natural-gas diffusion flames. Ph.D. Thesis, Delft University of Technology, 1995.

23. Pope, S.B., An explanation of the turbulent round-jet/plane-jet anomaly. AIAA J. 16 (1978) 279-281.

24. Pope, S.B., On the relationship between stochastic Lagrangian models of turbulence and second-moment closures. Phys. Fluids 6 (1994) 973-985.

25. Pope, S.B., Turbulent Flows. Cambridge University Press, Cambridge (2000).

26. Roekaerts, D., Reacting flows and probability density function methods. In: Launder, B. and Sandham, N. (eds.), Closure Strategies for Turbulent and Transitional Flows. Cambridge University Press, Cambridge (2002) pp. 47-101.

27. Speziale, C.G., Sarkar, S. and Gatski, T.B., Modelling the pressure-strain correlation of turbulence: An invariant system dynamic approach. J. Fluid Mech. 227 (1991) 245-272.

28. Wouters, H.A., Nooren, P.A., Peeters, T.W.J. and Roekaerts, D., Simulation of a bluff-bodystabilized diffusion flame using second-moment closure and Monte Carlo methods. Proc. Combust. Inst. 26 (1996) 246-265.

29. Wouters, H.A., Nooren, P.A., Peeters, T.W.J. and Roekaerts, D., Monte Carlo PDF modeling of a bluff-body stabilized methane diffusion flame. In: Hanjalic, K. and Peeters, T.W.J. (eds), Proceedings of 2nd International Symposium on Turbulence, Heat and Mass Transfer. Delft University of Technology (1997) pp. 389-398. 1997.

30. Wouters, H.A., Lagrangian models for turbulent reacting flows. Ph.D. Thesis, Delft University of Technology (1998).

31. Yang, S.L., Peschke, B.D. and Hanjalic, K., Second-moment closure model for IC engine flow simulation using KIVA code. J. Engrg. Gas Turbines and Power 122 (2000) 355-363. 